Stochastic and Deterministic Analysis of SIS Household Epidemics

\author{
Neal, Pete
}

2006

MIMS EPrint: 2006.47

Manchester Institute for Mathematical Sciences

School of Mathematics

The University of Manchester

\footnotetext{
Reports available from: http://eprints.maths.manchester.ac.uk/

And by contacting: The MIMS Secretary

School of Mathematics

The University of Manchester

Manchester, M13 9PL, UK
} 


\title{
Stochastic and Deterministic Analysis of Sis Household Epidemics
}

\author{
Peter Neal
}

First version: 26 September 2005

Research Report No. 8, 2005, Probability and Statistics Group School of Mathematics, The University of Manchester 


\title{
STOCHASTIC AND DETERMINISTIC ANALYSIS OF SIS HOUSE- HOLD EPIDEMICS
}

PETER NEAL, ${ }^{*}$ University of Manchester

\begin{abstract}
We analyse SIS epidemics amongst populations partitioned into households. The analysis considers both the stochastic and deterministic model and unlike previous analysis, we consider general infectious period distributions. For the deterministic model, we prove the existence of an endemic equilibrium for the epidemic if and only if the threshold parameter, $R_{*}>1$. Furthermore, by utilising Markov Chains we show that the total number of infectives converges to the endemic equilibrium as time $t \rightarrow \infty$. For the stochastic model, we prove a law of large numbers result for the convergence of the mean number of infectives per household in the stochastic model to the deterministic limit. This is followed by the derivation of a Gaussian limit process for the fluctuations of the stochastic model.

Keywords: Stochastic and deterministic models; SIS epidemics; Households model; Endemic equilibrium; Gaussian processes
\end{abstract}

AMS 2000 Subject Classification: Primary 92D30

Secondary 60G15; 37G35

\section{Introduction}

The household epidemic model which models the spread of an epidemic among a community of households has recently received considerable attention, see, for example, [5], [3], [2] and [4]. In all the above examples, closed population $S I R$ (susceptible $\rightarrow$ infective $\rightarrow$ removed) epidemics are considered and therefore endemic behaviour is not possible. The simplest epidemic model which can exhibit endemic behaviour is the closed population SIS epidemic model. That is, infectives at the end of their infectious period return to the susceptible state and therefore can be reinfected. The study of

\footnotetext{
* Postal address: School of Mathematics, University of Manchester, Sackville Street, Manchester, M60
} 1QD, UK 
homogeneously mixing closed population SIS epidemic models goes back to [16], see also [10] and [8] for stochastic analysis. However, it is only recently in [1] and [9] that the extension to a households epidemic model has been considered. The aim of the current work is to further study closed, population SIS household epidemic models.

In [1], Section 2 the initial stages of the epidemic process are considered, that is, where there is initially a few infectives in an otherwise susceptible population. In such circumstances, by considering a sequence of epidemics indexed by the total number of households, $n$, as $n \rightarrow \infty$ a branching process approximation for the epidemic can be derived. The branching process approximation can be used to answer the question of whether or not the SIS epidemic can exhibit endemic behaviour. The results of [1], Section 2 apply to a very general SIS epidemic model allowing for unequal sized households and general infectious periods. Therefore we shall focus on the endemic behaviour, or more specifically, the trajectory of the total number of infectives per household. In this respect, we extend the work of [1], Section 3 by taking a novel approach. In [1], Section 3, the special case of equal sized households (i.e. there exists $m \geq 2$ such that all households contain $m$ individuals) and exponentially distributed infectious periods is considered. This enables the derivation of a system of differential equations defining the deterministic SIS epidemic model, see [1], page 59, (10). The differential equations can then, in principle, be solved to determine the progress of the epidemic over time. Moreover, results of [11] and [12] can be utilised to prove convergence (suitably normalized) of the stochastic epidemic model to the deterministic limit and the derivation of a Gaussian limit process for the fluctuations of the epidemic model about the deterministic limit. Finally, in [1], for equal sized households of size 2 , the endemic behaviour of the deterministic model is considered and it is shown that if the initial proportion of the population which are infectious is positive then the distribution of the total number of infectives within the households converges to the endemic equilibrium, where it exists, as $t \rightarrow \infty$.

In [9], the $S I S$ household epidemic model is analysed using methods from statistical physics, namely self-consistent field methods. The analysis is essentially deterministic and they utilise the self-consistent field theory to consider the individual household epidemics as independent epidemics, subjected to a 'mean-field' global infection. We shall also utilise a construction which considers the individual household epidemics as 
conditionally independent given the total amount of global infection. However, our approach is rather different to [9] and we provide rigorous mathematical justification for our construction of the model.

The methods and results of [1] and [9] have two major drawbacks. Firstly, for $m \geq 1$ and $0 \leq i \leq m$, it is necessary to keep track of the total number of households of size $m$ with $i$ infectives. Therefore for $m \geq 1$, we require $m$ separate differential equations for households of size $m$, and so, for unequal sized household epidemics the system of differential equations rapidly grows in complexity. Secondly, it is required that each infectious period, the length of time from an individual becoming infected until they return to the susceptible state, is exponentially distributed. In order to surmount these problems we focus on a single quantity, the total number of infectives, or more precisely, the mean number of infectives per household. This then requires a radically different approach for analysing the model, a full description of which is given in Section 2 . Although our methods can be used with very general choices of infectious period, it is again necessary to restrict attention to exponentially distributed infectious periods to obtain explicit results. This is primarily due to difficulties in obtaining explicit expressions for the deterministic model for general choices of infectious period.

The paper is structured as follows. In Section 2, the household epidemic model is described in full detail. Since we are considering asymptotic results, we define a sequence of epidemic processes $\left\{E_{n}\right\}$, indexed by the total number of households, $n$, as $n \rightarrow \infty$. Then in Section 3, a weak law of large numbers result is derived for the convergence of the mean number of infectives per household to a deterministic limit. In Section 4, we study the deterministic limit in some detail for the case where the infectious periods are exponentially distributed. In particular, we extend [1], Theorem 3.1 to a very general household structure and thereby prove an associated conjecture (see, [1], page 64). These results establish necessary and sufficient conditions for the existence of an endemic equilibrium and show that, as $t \rightarrow \infty$, the epidemic converges to the endemic equilibrium, if it exists. Although the deterministic model can be described by a system of differential equations, our results are proved by utilising Markov birth-death processes and Markov Chains, via a coupling argument. Finally, in Section 5, we aim to extend the results of Section 3 by establishing a Gaussian limit process for the fluctuations of the stochastic model about the deterministic model. 
We are only able to do this for exponentially distributed infectious periods since we require an explicit expression for the deterministic model. However, in Theorem 5.1 we are able to obtain useful bounds for the fluctuations of the stochastic model for more general choices of infectious period. In particular, the bounds are of the same order of magnitude as those obtained from the Gaussian limit process.

\section{Model setup}

We consider a sequence of epidemic processes $\left\{E_{n}\right\}$ indexed by the total number of households, $n$, as $n \rightarrow \infty$. For $i \geq 1$, let $h_{i}$ denote the total number of individuals within household $i$. Note that we assume that for all $n \geq i$, the $i^{\text {th }}$ household in $E_{n}$ is of size $h_{i}$. (This assumption can easily be relaxed but is retained for clarity of exposition.) For $i \geq 1$, label the individuals in household $i,(i, 1),(i, 2), \ldots,\left(i, h_{i}\right)$. For fixed $n \geq 1$, the epidemic process is constructed as follows. An individual, $(i, j)$ say, that is, the $j^{t h}$ individual in household $i$ becomes infected at time $t$, say, and has infectious period $Q_{i j k}$, say, which is distributed according to an arbitrary, but specified, continuous non-negative distribution $Q$. The infectious period $Q_{i j k}$ is independent of all other infectious periods and individual $i$ is infectious in the time interval $\left[t, t+Q_{i j k}\right)$ before returning to the susceptible state at time $t+Q_{i j k}$. During its infectious period individual $(i, j)$ makes global infectious contacts at the points of a homogeneous Poisson point process with rate $\beta_{G}\left\{\frac{1}{n} \sum_{i=1}^{n} h_{i}\right\}$, the individual contacted by a global infectious contact is chosen uniformly at random from the entire population including individual $(i, j)$ itself. Also for $1 \leq k \leq h_{i}, k \neq j$, individual $(i, j)$, whilst infectious, makes infectious contacts with individual $(i, k)$ at the points of a homogeneous Poisson point process with rate $\beta_{L}$. Those individuals initially infectious at time $t=0$ are assumed to have independent and identically distributed infectious periods according to an arbitrary but specified continuous, non-negative distribution $\tilde{Q}$. Note that $\tilde{Q}$ and $Q$ can be distinct.

We consider the epidemic within individual households when each individual is exposed to a known global infectious pressure. For $i \geq 1, t \geq 0$ and $\mathbf{z}=\{z(s) ; s \geq 0\}$ let $\theta_{i}(t ; \mathbf{z})$ denote the total number of infectives within household $i$ at time $t$ given that each individual within household $i$ is contacted by global infections at the points 
of an inhomogeneous Poisson point process with rate $\beta_{G} z(s)$. Each individual within household $i$ then has infectious periods whose lengths are independently distributed according to $Q$ (initial infectious periods distributed according to $\tilde{Q}$ ). An individual, whilst infectious, makes local infectious contacts with a given individual within household $i$ at the points of a homogeneous Poisson point process with rate $\beta_{L}$. The most important fact to note is that for $i \neq j$, and $s, t \geq 0, \theta_{i}(t ; \mathbf{z})$ and $\theta_{j}(s ; \mathbf{z})$ are independent.

Therefore if we let $\tilde{X}_{n}(t)=n X_{n}(t)$ denote the total number of individuals infectious in $E_{n}$ at time $t$, then $X_{n}(t)=\frac{1}{n} \sum_{i=1}^{n} \theta_{i}\left(t ; \mathbf{X}_{n}\right)$. The key point is that by this construction the epidemics within distinct households are conditionally independent, given $\mathbf{X}_{n}=\left\{X_{n}(s) ; s \geq 0\right\}$. Clearly, this introduces an explicit dependence upon the entire past history of the epidemic for considering $X_{n}(t)$, however, it transpires that this will not be problematic.

For $k \geq 1$ and $0 \leq l \leq k$, let $a_{k l}^{n}$ denote the total number of households of size $k$ within $E_{n}$ which contain $l$ initial infectives. For $k \geq 1$, let $\omega_{k}^{n}=\frac{1}{n} \sum_{l=0}^{k} a_{k l}^{n}$ denote the proportion of households of size $k$ and for $0 \leq l \leq k$, let $\zeta_{k l}^{n}=\frac{a_{k l}^{n}}{n \omega_{k}^{n}}$. Suppose that for $k \geq 1$ and $0 \leq l \leq k, \omega_{k}^{n} \rightarrow \omega_{k}$ and $\zeta_{k l}^{n} \rightarrow \zeta_{k l}$ as $n \rightarrow \infty$ with $\sum_{k=1}^{\infty} \omega_{k}=1$ and $\sum_{l=0}^{k} \zeta_{k l}=1$. Finally, let $H$ be a positive discrete random variable with $\mathbb{P}(H=k)=\omega_{k}$ $(k \geq 1)$. Thus $H$ denotes the limiting probability distribution for household size and throughout we will specify conditions on the household size distribution in terms of $H$.

For $k \geq 1,0 \leq l \leq k, t \geq 0$ and $\mathbf{z}=\{z(s) ; s \geq 0\}$, let $\chi_{k l}(t ; \mathbf{z})$ denote the total number of infectives at time $t$ within a household of size $k$ with $l$ initial infectives that each individual within the household is contacted by global infections at the points of an inhomogeneous Poisson point process with rate $\beta_{G} z(s)$. Thus for $i \geq 1$, if $h_{i}=k$ and $\theta_{i}(0 ; \mathbf{z})=l$, then for all $t \geq 0, \theta_{i}(t ; \mathbf{z}) \stackrel{D}{=} \chi_{k l}(t ; \mathbf{z})$.

Let $x(0)=\sum_{k=1}^{\infty} \omega_{k} \sum_{l=0}^{k} l \zeta_{k l}$ and for $t>0$

$$
x(t)=\sum_{k=1}^{\infty} \omega_{k} \sum_{l=0}^{k} \zeta_{k l} \mathbb{E}\left[\chi_{k l}(t ; \mathbf{x})\right]
$$

Thus $\{x(s) ; s \geq 0\}$ is the deterministic equivalent of the limiting stochastic model described above. In Section 4, we shall analyse (2.1) in detail for the case $Q \sim \operatorname{Exp}(\gamma)$. 


\section{Law of large numbers analysis}

In this section, we show that if $\mathbb{E}\left[H^{2}\right]<\infty$, then for any $T>0$,

$$
\sup _{0 \leq s \leq T}\left|X_{n}(s)-x(s)\right| \stackrel{p}{\longrightarrow} 0 \quad \text { as } n \rightarrow \infty .
$$

We begin by stating the following useful proposition.

Proposition 3.1. For any two inhomogeneous Poisson point processes $\eta_{1}$ and $\eta_{2}$ with rates $\alpha_{1}(s)$ and $\alpha_{2}(s)$, respectively. There exists a coupling such that for any $0 \leq t<T$,

$$
\eta_{1}[0, t]=\eta_{2}[0, t]
$$

where $T$ is a non-negative random variable with cumulative distribution function

$$
\mathbb{P}(T \leq t)=1-\exp \left(-\int_{0}^{t}\left|\alpha_{1}(s)-\alpha_{2}(s)\right| d s\right) .
$$

Let $x_{n}(0)=\sum_{k=1}^{\infty} \omega_{k}^{n} \sum_{l=0}^{k} l \zeta_{k l}^{n}$ and for $t>0$,

$$
x_{n}(t)=\sum_{k=1}^{\infty} \omega_{k}^{n} \sum_{l=0}^{k} \zeta_{k l}^{n} \mathbb{E}\left[\chi_{k l}\left(t ; \mathbf{x}_{n}\right)\right] \equiv \frac{1}{n} \sum_{i=1}^{n} \mathbb{E}\left[\theta_{i}^{n}\left(t ; \mathbf{x}_{n}\right)\right] .
$$

Thus $\left\{x_{n}(s) ; s \geq 0\right\}$ is the deterministic equivalent of $E_{n}$. Furthermore, we have the following lemma linking $x_{n}(\cdot)$ and $x(\cdot)$.

Lemma 3.1. Suppose that $\sum_{k=1}^{\infty} k \sum_{l=0}^{k}\left|\omega_{k}^{n} \zeta_{k l}^{n}-\omega_{k} \zeta_{k l}\right| \rightarrow 0$ as $n \rightarrow \infty$, then for any $T>0$,

$$
\sup _{0 \leq s \leq T}\left|x_{n}(s)-x(s)\right| \rightarrow 0 \quad \text { as } n \rightarrow \infty .
$$

Proof. For $k \leq 1$ and $0 \leq l \leq k$,

$$
\sup _{0 \leq s \leq T} \mathbb{E}\left[\left|\chi_{k l}(s ; \mathbf{x})-\chi_{k l}\left(s ; \mathbf{x}_{n}\right)\right|\right] \leq k \mathbb{P}\left(\sup _{0 \leq s \leq T}\left|\chi_{k l}(s ; \mathbf{x})-\chi_{k l}\left(s ; \mathbf{x}_{n}\right)\right| \neq 0\right) .
$$

By Proposition 3.1, there exists a random variable $T_{n}^{k}$ with

$$
\mathbb{P}\left(T_{n}^{k} \leq T\right)=1-\exp \left(-k \beta_{G} \int_{0}^{T}\left|x(s)-x_{n}(s)\right| d s\right)
$$

such that the inhomogeneous Poisson point processes $\eta_{k}$ and $\eta_{k}^{n}$ with rates $k \beta_{G} x(s)$ and $k \beta_{G} x_{n}(s)$, respectively, can be coupled, so that, for any $0 \leq t<T_{n}^{k}$,

$$
\eta_{k}[0, t]=\eta_{k}^{n}[0, t] .
$$


Therefore, we have that

$$
\begin{aligned}
\mathbb{P}\left(\sup _{0 \leq s \leq t}\left|\chi_{k l}(t ; \mathbf{x})-\chi_{k l}\left(t ; \mathbf{x}_{n}\right)\right| \neq 0\right) & \leq \mathbb{P}\left(T_{n}^{k} \leq T\right) \\
& \leq k \beta_{G} \int_{0}^{T}\left|x(s)-x_{n}(s)\right| d s
\end{aligned}
$$

Thus, by (3.3)

$$
\begin{aligned}
& \sup _{0 \leq s \leq T}\left|x(s)-x_{n}(s)\right| \\
\leq & \sum_{k=1}^{\infty} \sum_{l=0}^{k} \sup _{0 \leq s \leq T}\left|\omega_{k} \zeta_{k l} \mathbb{E}\left[\chi_{k l}(s ; \mathbf{x})\right]-\omega_{k}^{n} \zeta_{k l}^{n} \mathbb{E}\left[\chi_{k l}\left(s ; \mathbf{x}_{n}\right)\right]\right| \\
\leq & \sum_{k=1}^{\infty} k \sum_{l=0}^{k}\left|\omega_{k} \zeta_{k l}-\omega_{k}^{n} \zeta_{k l}^{n}\right|+\sum_{k=1}^{\infty} \omega_{k} \sum_{l=0}^{k} \zeta_{k l} \mathbb{E}\left[\sup _{0 \leq s \leq T}\left|\chi_{k l}(s ; \mathbf{x})-\chi_{k l}\left(s ; \mathbf{x}_{n}\right)\right|\right] \\
\leq & \sum_{k=1}^{\infty} k \sum_{l=0}^{k}\left|\omega_{k} \zeta_{k l}-\omega_{k}^{n} \zeta_{k l}^{n}\right|+\sum_{k=1}^{\infty}\left\{\omega_{k} k^{2} \beta_{G} \int_{0}^{T}\left|x(s)-x_{n}(s)\right| d s\right\} \\
\leq & \sum_{k=1}^{\infty} k \sum_{l=0}^{k}\left|\omega_{k} \zeta_{k l}-\omega_{k}^{n} \zeta_{k l}^{n}\right|+\beta_{G} \mathbb{E}\left[H^{2}\right] \int_{0}^{T} \sup _{0 \leq u \leq s}\left|x(u)-x_{n}(u)\right| d s
\end{aligned}
$$

Therefore it follows by Gronwall's inequality that,

$$
\sup _{0 \leq s \leq T}\left|x(s)-x_{n}(s)\right| \leq\left\{\sum_{k=1}^{\infty} k \sum_{l=0}^{k}\left|\omega_{k}^{n} \zeta_{k l}^{n}-\omega_{k} \zeta_{k l}\right|\right\} \exp \left(\beta_{G} T \mathbb{E}\left[H^{2}\right]\right)
$$

and the lemma follows since the righthand-side of (3.4) converges to 0 as $n \rightarrow \infty$.

Note that, for all $t \geq 0$,

$$
\begin{aligned}
X_{n}(t)-x_{n}(t)=\frac{1}{n} \sum_{i=1}^{n}\left\{\theta_{i}(t\right. & \left.\left.; \mathbf{X}_{n}\right)-\mathbb{E}\left[\theta_{i}\left(t ; \mathbf{X}_{n}\right) \mid \mathbf{X}_{n}\right]\right\} \\
& +\frac{1}{n} \sum_{i=1}^{n}\left\{\mathbb{E}\left[\theta_{i}\left(t ; \mathbf{X}_{n}\right) \mid \mathbf{X}_{n}\right]-\mathbb{E}\left[\theta_{i}\left(t ; \mathbf{x}_{n}\right)\right]\right\} .
\end{aligned}
$$

We shall make use of a sequence of immigration-death processes $\left\{I_{n}\right\}_{n \geq 1}$, to assist in proving that

$$
\sup _{0 \leq s \leq T}\left|\frac{1}{n} \sum_{i=1}^{n}\left\{\theta_{i}\left(s ; \mathbf{X}_{n}\right)-\mathbb{E}\left[\theta_{i}\left(s ; \mathbf{X}_{n}\right) \mid \mathbf{X}_{n}\right]\right\}\right| \stackrel{p}{\longrightarrow} 0 \quad \text { as } n \rightarrow \infty .
$$

For $n \geq 1$, suppose that immigrations occur at the points of a homogeneous Poisson point process with rate

$$
n K_{n}=\frac{1}{4} \frac{\beta_{G}}{n}\left\{\sum_{i=1}^{n} h_{i}\right\}^{2}+\beta_{L} \sum_{i=1}^{n} \frac{h_{i}^{2}}{4}=n \frac{1}{4}\left\{\beta_{G}\left(\frac{1}{n} \sum_{i=1}^{n} h_{i}\right)^{2}+\beta_{L} \frac{1}{n} \sum_{i=1}^{n} h_{i}^{2}\right\} .
$$


Note that

$$
K_{n} \rightarrow K=\frac{1}{4}\left\{\beta_{G} \mathbb{E}[H]^{2}+\beta_{L} \mathbb{E}\left[H^{2}\right]\right\} \quad \text { as } n \rightarrow \infty .
$$

Each immigrant upon immigrating into the population has an independent and identically distributed lifetime according to $Q$. That is, if individual, $i$ say, with lifetime $Q_{i}$, immigrates into the population at time, $t$ say, then individual $i$ dies at time $t+Q_{i}$.

Further suppose that, for $n \geq 1$, the process $I_{n}$ starts at time $t=0$ with $r_{n}$ initial individuals in the population. Suppose that there exists $r \geq 0$ such that $\frac{r_{n}}{n} \rightarrow r$ as $n \rightarrow \infty$ and the initial individuals have independent and identically distributed death times $\tilde{Q}_{1}, \tilde{Q}_{2}, \ldots, \tilde{Q}_{r_{n}}$, respectively. For $k \geq 1$, let $\left(t_{k}^{n}, Q_{k}, U_{k}\right)$ denote the immigration time and length of the lifetime, respectively, of the $k^{\text {th }}$ immigrant in $I_{n}$ with $U_{k}$ denoting a uniform random variable on $[0,1]$ which plays no part in the immigration-death process but is instrumental in coupling the epidemic process to the immigration-death process. It is assumed that the random variables $\left(Q_{k}, U_{k}\right)$ are independent and identically distributed with $Q_{k}$ and $U_{k}$ independent.

For $n \geq 1$ and $t \geq 0$, let

$$
\begin{aligned}
& A_{n}^{I}(t)=\left\{\left(t_{k}^{n}, Q_{k}, U_{k}\right): t_{k}^{n} \leq t\right\} \\
& A_{n}^{D}(t)=\left\{\left(t_{k}^{n}, Q_{k}, U_{k}\right): t_{k}^{n}+Q_{k} \leq t\right\} \cup\left\{\tilde{Q}_{k}: \tilde{Q}_{k} \leq t\right\} .
\end{aligned}
$$

Therefore the sets $A_{n}^{I}(t)$ and $A_{n}^{D}(t)$ denote those individuals whom have immigrated into $I_{n}$ and died in $I_{n}$, respectively, up to and including time $t$. For $n \geq 1$ and $t \geq 0$, let $\tilde{Z}_{n}^{I}(t)=n Z_{n}^{I}(t)$ and $\tilde{Z}_{n}^{D}(t)=n Z_{n}^{D}(t)$ denote the cardinalities of $A_{n}^{I}(t)$ and $A_{n}^{D}(t)$, respectively. Hence $\tilde{Z}_{n}^{I}(t)$ and $\tilde{Z}_{n}^{D}(t)$ denote the total number of immigrations and deaths, respectively, in $I_{n}$ up to time $t$.

The key point in utilising $I_{n}$, is that in the epidemic $E_{n}$ infections occur at the points of an inhomogeneous Poisson point process with rate $n \lambda_{n}(t)$ given by

$$
\frac{\beta_{G}}{n}\left\{\sum_{i=1}^{n} \theta_{i}\left(t ; \mathbf{X}_{n}\right)\right\}\left\{\sum_{i=1}^{n}\left(h_{i}-\theta_{i}\left(t ; \mathbf{X}_{n}\right)\right)\right\}+\beta_{L} \sum_{i=1}^{n} \theta_{i}\left(t ; \mathbf{X}_{n}\right)\left(h_{i}-\theta_{i}\left(t ; \mathbf{X}_{n}\right)\right)(t) .
$$

Hence for all $t \geq 0, \lambda_{n}(t) \leq K_{n}$. Thus for $n \geq 1$, the epidemic $E_{n}$ can be coupled to the immigration process $I_{n}$ as follows. Suppose that $r_{n}=n X_{n}(0)\left(=n x_{n}(0)\right)$, then let the initial infectives in $E_{n}$ have (remaining) infectious periods $\tilde{Q}_{1}, \tilde{Q}_{2}, \ldots, \tilde{Q}_{r_{n}}$, respectively. Thus for every removal of an initial infective in $E_{n}$ there will be a 
corresponding death of an initial individual in $I_{n}$. Now for $k \geq 1$, consider the $k^{\text {th }}$ immigration in $I_{n}$, then if $U_{k} \leq \lambda_{n}\left(t_{k}^{n}\right) / K_{n}$ an infection occurs in $E_{n}$ and the infectious period of the infective is $Q_{k}$. The individual infected can then be chosen from amongst the susceptibles according to the correct conditional distribution. Thus we have a coupling such that for all $n \geq 1$ and $0 \leq s \leq t$,

$$
\left|X_{n}(t)-X_{n}(s)\right| \leq\left\{Z_{n}^{I}(t)-Z_{n}^{I}(s)\right\}+\left\{Z_{n}^{D}(t)-Z_{n}^{D}(s)\right\},
$$

where $Z_{n}^{I}(\cdot)$ and $Z_{n}^{D}(\cdot)$ are more convenient to analyse than $X_{n}(\cdot)$.

Note that, for all $t \geq 0$,

$$
\begin{aligned}
& Z_{n}^{I}(t) \sim \frac{1}{n} \operatorname{Po}\left(n K_{n} t\right) \\
& Z_{n}^{D}(t) \sim \frac{1}{n}\left\{\operatorname{Bin}\left(m_{n}, \tilde{F}(t)\right)+\operatorname{Po}\left(n K_{n}(t F(t))\right\}\right.
\end{aligned}
$$

where for $s \geq 0, \tilde{F}(s)$ and $F(s)$ denote the cumulative distribution functions of $\tilde{Q}$ and $Q$, respectively.

Lemma 3.2. For all $T>0$,

$$
\sup _{0 \leq s \leq T}\left|\frac{1}{n} \sum_{i=1}^{n}\left\{\theta_{i}\left(s ; \mathbf{X}_{n}\right)-\mathbb{E}\left[\theta_{i}\left(s ; \mathbf{X}_{n}\right) \mid \mathbf{X}_{n}\right]\right\}\right| \stackrel{p}{\longrightarrow} 0 \quad \text { as } n \rightarrow \infty .
$$

Proof. Firstly note that using (3.6) and Chebychev's inequality, it is trivial to show that, for all $s \geq 0$,

$$
\left|Z_{n}^{I}(s)-\mathbb{E}\left[Z_{n}^{I}(s)\right]\right| \stackrel{p}{\longrightarrow} 0 \quad \text { and } \quad\left|Z_{n}^{D}(s)-\mathbb{E}\left[Z_{n}^{D}(s)\right]\right| \stackrel{p}{\longrightarrow} 0 \quad \text { as } n \rightarrow \infty .
$$

Furthermore, since the $\left\{\theta_{i}\left(s ; \mathbf{X}_{n}\right)\right\}$ 's are conditionally independent given $\mathbf{X}_{n}$, it follows by Chebychev's inequality (c.f. [14], Lemma 5.4), that for any $s \geq 0$ and $\epsilon>0$,

$$
\begin{aligned}
& \mathbb{P}\left(\left|\frac{1}{n} \sum_{i=1}^{n}\left\{\theta_{i}\left(s ; \mathbf{X}_{n}\right)-\mathbb{E}\left[\theta_{i}\left(s ; \mathbf{X}_{n}\right) \mid \mathbf{X}_{n}\right]\right\}\right|>\epsilon\right) \\
\leq & \frac{1}{\epsilon^{2}} \frac{1}{n^{2}} \mathbb{E}\left[\operatorname{var}\left(\sum_{i=1}^{n} \theta_{i}\left(s ; \mathbf{X}_{n}\right) \mid \mathbf{X}_{n}\right)\right] \\
\leq & \frac{1}{\epsilon^{2}} \frac{1}{n^{2}} \sum_{i=1}^{n} h_{i}^{2}=\frac{1}{\epsilon^{2} n}\left\{\sum_{k=1}^{\infty} k^{2} \omega_{k}^{n}\right\} \\
\rightarrow & 0 \quad \text { as } n \rightarrow \infty .
\end{aligned}
$$

Fix $T \geq 0$ and $\epsilon>0$. For $0 \leq s \leq T$, let $z^{I}(s)=K s$ and $z^{D}(s)=x(0) \tilde{F}(s)+$ $K s F(s)$. Note that $z^{I}(T), z^{D}(T)<\infty$ and $z^{I}(s)$ and $z^{D}(s)$ are non-decreasing in 
s. Therefore there exists $r \in \mathbb{N}$ and $0=u_{0}<u_{1}<\ldots<u_{r}=T$ such that if $B_{k}=\left\{s: u_{k} \leq s \leq u_{k+1}\right\}(0 \leq k \leq r-1), \max _{s, s^{\prime} \in B_{k}}\left|z^{I}(s)-z^{I}\left(s^{\prime}\right)\right|<\frac{\epsilon}{9}$ and $\max _{s, s^{\prime} \in B_{k}}\left|z^{D}(s)-z^{D}\left(s^{\prime}\right)\right|<\frac{\epsilon}{9}$, for all $(0 \leq k \leq r-1)$. Therefore since for all $0 \leq s \leq T, \mathbb{E}\left[Z_{n}^{I}(s)\right] \rightarrow z^{I}(s)$ and $\mathbb{E}\left[Z_{n}^{D}(s)\right] \rightarrow z^{D}(s)$ as $n \rightarrow \infty$, it follows that

$$
\begin{aligned}
\lim _{n \rightarrow \infty} \mathbb{P}\left(\left|Z_{n}^{I}(s)-z^{I}(s)\right|<\frac{\epsilon}{9}, s \in H_{r}\right) & =1, \\
\lim _{n \rightarrow \infty} \mathbb{P}\left(\left|Z_{n}^{D}(s)-z^{D}(s)\right|<\frac{\epsilon}{9}, s \in H_{r}\right) & =1, \\
\lim _{n \rightarrow \infty} \mathbb{P}\left(\left|\frac{1}{n} \sum_{i=1}^{n}\left\{\theta_{i}\left(s ; \mathbf{X}_{n}\right)-\mathbb{E}\left[\theta_{i}\left(s ; \mathbf{X}_{n}\right) \mid \mathbf{X}_{n}\right]\right\}\right|<\frac{\epsilon}{9}, s \in H_{r}\right) & =1,
\end{aligned}
$$

where $H_{r}=\left\{u_{0}, u_{1}, \ldots, u_{r}\right\}$.

Now by using (3.6) and the triangle inequality, it is straightforward to show that, for all $0 \leq k \leq r-1$ and $u_{k} \leq s \leq u_{k+1}$,

$$
\begin{aligned}
& \left|\frac{1}{n} \sum_{i=1}^{n}\left\{\theta_{i}\left(s ; \mathbf{X}_{n}\right)-\mathbb{E}\left[\theta_{i}\left(s ; \mathbf{X}_{n}\right) \mid \mathbf{X}_{n}\right]\right\}\right| \\
\leq & \left|Z_{n}^{I}\left(u_{k+1}\right)-z^{I}\left(u_{k+1}\right)\right|+\left|Z_{n}^{I}\left(u_{k}\right)-z^{I}\left(u_{k}\right)\right|+\left|Z_{n}^{D}\left(u_{k+1}\right)-z^{D}\left(u_{k+1}\right)\right| \\
& +\left|Z_{n}^{D}\left(u_{k}\right)-z^{D}\left(u_{k}\right)\right|+\left|\frac{1}{n} \sum_{i=1}^{n}\left\{\theta_{i}^{n}\left(u_{k} ; \mathbf{X}_{n}\right)-\mathbb{E}\left[\theta_{i}^{n}\left(u_{k} ; \mathbf{X}_{n}\right) \mid \mathbf{X}_{n}\right]\right\}\right| \\
& +2\left(z^{I}\left(u_{k+1}\right)-z^{I}\left(u_{k}\right)\right)+2\left(z^{D}\left(u_{k+1}\right)-z^{D}\left(u_{k}\right)\right) .
\end{aligned}
$$

Thus by considering each $B_{k}$ separately, it follows that, if for all $u \in H_{r}$,

$$
\begin{aligned}
\left|Z_{n}^{I}(u)-z^{I}(u)\right| & <\frac{\epsilon}{9} \\
\left|Z_{n}^{D}(u)-z^{D}(u)\right| & <\frac{\epsilon}{9} \\
\left|\frac{1}{n} \sum_{i=1}^{n}\left\{\theta_{i}\left(u ; \mathbf{X}_{n}\right)-\mathbb{E}\left[\theta_{i}\left(u ; \mathbf{X}_{n}\right) \mid \mathbf{X}_{n}\right]\right\}\right| & <\frac{\epsilon}{9}
\end{aligned}
$$

then

$$
\sup _{0 \leq s \leq T}\left|\frac{1}{n} \sum_{i=1}^{n}\left\{\theta_{i}\left(s ; \mathbf{X}_{n}\right)-\mathbb{E}\left[\theta_{i}\left(s ; \mathbf{X}_{n}\right) \mid \mathbf{X}_{n}\right]\right\}\right|<\epsilon,
$$

and the lemma follows using (3.7), (3.8) and (3.9).

Thus we are now ready to prove (3.1).

Theorem 3.1. For all $T>0$,

$$
\sup _{0 \leq s \leq T}\left|X_{n}(s)-x(s)\right| \stackrel{p}{\longrightarrow} 0 \quad \text { as } n \rightarrow \infty
$$


Proof. By Lemma 3.1 and the triangle inequality, it is sufficient to show that

$$
\sup _{0 \leq s \leq T}\left|X_{n}(s)-x_{n}(s)\right| \stackrel{p}{\longrightarrow} 0 \quad \text { as } n \rightarrow \infty .
$$

Note that by the triangle inequality,

$$
\sup _{0 \leq s \leq T}\left|X_{n}(s)-x_{n}(s)\right| \leq A_{n}(T)+B_{n}(T)
$$

where for $t \geq 0$,

$$
\begin{aligned}
& A_{n}(t)=\sup _{0 \leq s \leq t}\left|\frac{1}{n} \sum_{i=1}^{n}\left\{\theta_{i}\left(t ; \mathbf{X}_{n}\right)-\mathbb{E}\left[\theta_{i}\left(t ; \mathbf{X}_{n}\right) \mid \mathbf{X}_{n}\right]\right\}\right| \\
& B_{n}(t)=\sup _{0 \leq s \leq t}\left|\frac{1}{n} \sum_{i=1}^{n}\left\{\mathbb{E}\left[\theta_{i}\left(t ; \mathbf{X}_{n}\right) \mid \mathbf{X}_{n}\right]-\mathbb{E}\left[\theta_{i}\left(t ; \mathbf{x}_{n}\right)\right]\right\}\right|
\end{aligned}
$$

Note that

$$
B_{n}(t)=\sup _{0 \leq s \leq t}\left|\sum_{k=1}^{\infty} \omega_{k}^{n} \sum_{l=0}^{k} \zeta_{k l}^{n}\left\{\mathbb{E}\left[\chi_{k l}\left(s ; \mathbf{X}_{n}\right) \mid \mathbf{X}_{n}\right]-\mathbb{E}\left[\chi_{k l}\left(s ; \mathbf{x}_{n}\right)\right]\right\}\right| .
$$

Therefore by similar arguments to those used in Lemma 3.1, for $t \geq 0$,

$$
B_{n}(t) \leq\left\{\sum_{k=1}^{\infty} k^{2} \omega_{k}^{n}\right\} \beta_{G} \int_{0}^{t} \sup _{0 \leq u \leq s}\left|X_{n}(u)-x_{n}(u)\right| d s
$$

Thus by using (3.11) and applying Gronwall's inequality to (3.10), we have that for $T \geq 0$,

$$
\sup _{0 \leq s \leq T}\left|X_{n}(s)-x_{n}(s)\right| \leq A_{n}(T) \exp \left(\left\{\sum_{k=1}^{\infty} k^{2} \omega_{k}^{n}\right\} \beta_{G} T\right)
$$

and the lemma follows, since by Lemma 3.2 the righthand-side of (3.12) converges in probability to 0 as $n \rightarrow \infty$.

\section{Deterministic model}

In order to study the deterministic model $x(\cdot)$, we restrict attention to the case $Q \sim \operatorname{Exp}(\gamma)$, and we can then use Markov Chains to analyse, $x(\cdot)$. In particular, we prove a generalisation of [1], Theorem 3.1, and hence, prove a related conjecture (see, [1], page 64 ) under the weak condition that $\mathbb{E}[H]<\infty$.

We begin by considering a household of size $m$, for some $m \geq 1$. Suppose that each individual within the household is contacted by global infections at the points of an 
inhomogeneous Poisson point process with rate $\beta_{G} z(t)$, for some non-negative function $z(\cdot)$.

For $s \geq 0$, let $G_{m}(u)$ denote a $(m+1) \times(m+1)$ matrix with typical entries $g_{i j}^{m}(u)$ $(0 \leq i, j \leq m)$, where

$$
\begin{array}{ll}
g_{i(i+1)}^{m}(u)=(m-i) \beta_{G} u+(m-i) i \beta_{L} & (0 \leq i \leq m-1) \\
g_{i(i-1)}^{m}(u)=i \gamma & (1 \leq i \leq m) \\
g_{i j}^{m}(u)=0 & (|i-j| \geq 2 ; 0 \leq i, j \leq m) \\
g_{i i}^{m}(u)=-\sum_{j \neq i} g_{i j}^{m}(u) & (0 \leq i \leq m)
\end{array}
$$

and hence, $g_{i j}^{m}(u)$ is the infinitesimal transition rate from $i$ to $j$ infectives given that there are $u$ units of global infectious pressure. We shall use $G_{m}(u)$ to construct a time inhomogeneous Markov chain to study the total number of infectives within the household at any given point in time.

For $0 \leq a \leq b$, let $S_{m}(a, b ; \mathbf{z})=\exp \left(\int_{a}^{b} G_{m}(z(s)) d s\right)$ with typical entries $s_{i j}^{m}(a, b ; \mathbf{z})$ $(0 \leq i, j \leq m)$. Thus for $0 \leq i, j \leq m, s_{i j}^{m}(a, b ; \mathbf{z})$ denotes the probability that there are $j$ infectives within the household at time $b$ given that there were $i$ infectives at time $a$.

Turning attention to $x(\cdot)$, we note that, for $t \geq 0, x(t)$ solves the equation

$$
x(t)=\sum_{k=1}^{\infty} \omega_{k} \mathbf{k}\left\{\boldsymbol{\zeta}_{k} S_{k}(0, t ; \mathbf{x})\right\}^{T}
$$

with $x(0)=\sum_{k=1}^{\infty} \omega_{k} \mathbf{k} \boldsymbol{\zeta}_{k}^{T}$.

In order to analyse $x(\cdot)$ and, in particular, $\lim _{t \rightarrow \infty} x(t)$, we need to introduce some basic notation and results. For $k \geq 1$, let

$$
R_{*}^{(k)}=\frac{\beta_{G}}{\gamma} \mathbb{E}[H] \frac{(k-1) !}{\rho^{k-1}} \sum_{i=0}^{k-1} \frac{\rho^{i}}{i !}
$$

and

$$
R_{*}=\sum_{k=1}^{\infty} k \omega_{k} R_{*}^{(k)} / \mathbb{E}[H]=\sum_{k=1}^{\infty}\left\{\omega_{k} \frac{\beta_{G}}{\gamma} \frac{k !}{\rho^{k-1}} \sum_{i=0}^{k-1} \frac{\rho^{i}}{i !}\right\}
$$

where $\rho=\frac{\gamma}{\beta_{L}}$. The quantity $R_{*}$ plays a vital role in both the stochastic and deterministic analysis of the epidemic model. In this situation, $R_{*}^{(k)}$ denotes the mean number of global infectious contacts (births) emanating from the epidemic within a household of size $k$ (individual in the branching process), where initially there is one infective within the household and there are no global infections into the household. 
Then for the stochastic model, it is shown in [1] that if $R_{*} \leq 1$, the approximating branching process goes extinct, almost surely, and the epidemic also dies out. Whereas if $R_{*}>1$, there is a non-zero probability that the branching process does not go extinct corresponding to the epidemic taking off. The deterministic model is analysed below. We show that if $R_{*} \leq 1$, there exists only one equilibrium point $x^{*}=0$ and this point is an attractor, that is, for all $x(0) \geq 0, x(t) \rightarrow x^{*}(\equiv 0)$ as $t \rightarrow \infty$. For $R_{*}>1$, there are two equilibrium points, $x^{*}=0$ and $s^{*}>0$. Then $x^{*}$ is an unstable equilibrium point whilst $s^{*}$ is an attractor, in that, for any configuration of initial infectives such that $x(0)>0, x(t) \rightarrow s^{*}$ as $t \rightarrow \infty$. Analysis of the distribution of the total number of infectives within the different sized households, in equilibrium, is also given.

The first step is to consider for $m \geq 1$ and $s \geq 0$, time-homogeneous Markov birthdeath processes with transition matrices, $G_{m}(s)$, corresponding to epidemics within households of size $m$ subjected to a constant global infectious pressure, $s$. Let $\boldsymbol{\pi}_{m}(s)$ denote the stationary distribution of the birth-death process, hence

$$
\mathbf{0}=\boldsymbol{\pi}_{m}(s) G_{m}(s)
$$

and if, for $t \geq 0, \tilde{S}_{m}(t ; s)=\exp \left(t G_{m}(s)\right)$ then, for all $t \geq 0$,

$$
\boldsymbol{\pi}_{m}(s)=\boldsymbol{\pi}_{m}(s) \tilde{S}_{m}(t ; s) .
$$

For any $m \geq 1, \mathbf{a}, \mathbf{b} \in \mathbb{R}^{m+1}$, let $\mathbf{a}<\mathbf{b}(\mathbf{a} \leq \mathbf{b})$ if $\sum_{i=0}^{m} a_{i}=\sum_{i=0}^{m} b_{i}$ and for all $1 \leq k \leq m, \sum_{i=k}^{m} a_{i}<\sum_{i=k}^{m} b_{i}\left(\sum_{i=k}^{m} a_{i} \leq \sum_{i=k}^{m} b_{i}\right)$. It is trivial to prove that for $s<s^{\prime}, \boldsymbol{\pi}_{m}(s)<\boldsymbol{\pi}_{m}\left(s^{\prime}\right)$ since for $s>0$,

$$
\pi_{m}^{0}(s)=\left\{1+\sum_{k=1}^{m} \prod_{i=1}^{k} \frac{(m+1-i)\left(\beta_{G} s+(i-1) \beta_{L}\right)}{i \gamma}\right\}^{-1}
$$

and for $1 \leq k \leq m$,

$$
\pi_{m}^{k}(s)=\frac{(m+1-k)\left(\beta_{G} s+(k-1) \beta_{L}\right)}{k \gamma} \pi_{m}^{k-1}(s) .
$$

For $s \geq 0$, let $\mu_{m}(s)=\sum_{i=1}^{m} i \pi_{m}^{i}(s)$ denote the mean number of infectives, in stationarity, within a household of size $m$ given that the household is subjected to a constant global infectious pressure, $s$, and let $\mu(s)=\sum_{k=1}^{\infty} \omega_{k} \mu_{k}(s)$.

We shall consider $\mu(s)$ and, in particular, the solutions of $\mu(s)=s$ after the following preliminary Lemma. 
Lemma 4.1. For $m \geq 1$ and $a_{i}, b_{i} \geq 0(0 \leq i \leq m)$, suppose that $f_{m}(x)=\sum_{i=0}^{m} a_{i} x^{i}$ and $g_{m}(x)=\sum_{i=0}^{m} b_{i} x^{i}$. Suppose that for $0 \leq j<k \leq m, \frac{a_{j}}{b_{j}}<\frac{a_{k}}{b_{k}}$, then for all $x \geq 0$,

$$
\frac{d}{d x}\left(\frac{f_{m}(x)}{g_{m}(x)}\right)>0
$$

and

$$
\frac{d^{2}}{d x^{2}}\left(\frac{f_{m}(x)}{g_{m}(x)}\right)<0 .
$$

Proof. The lemma trivially holds for $m=1$ and can then be proved using induction upon $m$ by considering $\frac{d}{d x}\left(\frac{a_{m} x^{m}+f_{m-1}(x)}{b_{m} x^{m}+g_{m-1}(x)}\right)$ and $\frac{d^{2}}{d x^{2}}\left(\frac{a_{m} x^{m}+f_{m-1}(x)}{b_{m} x^{m}+g_{m-1}(x)}\right)$.

An immediate consequence of Lemma 4.1 is the following corollary.

Corollary 4.1. For all $m \geq 1, \mu_{m}(s)$ is a concave function with for all $s \geq 0$,

$$
\frac{d}{d s} \mu_{m}(s)>0 \quad \text { and } \quad \frac{d^{2}}{d s^{2}} \mu_{m}(s)<0 .
$$

Hence, $\mu(s)$ is a concave function with for all $s \geq 0$,

$$
\frac{d}{d s} \mu(s)>0 \quad \text { and } \quad \frac{d^{2}}{d s^{2}} \mu(s)<0 .
$$

Note that $\mu(0)=0$ and $\mu(\infty)=\mathbb{E}[H]$. Thus from Corollary 4.1 there exists at most one $s>0$ such that $\mu(s)=s$. Furthermore, such a solution will only exist if $\left.\frac{d}{d s} \mu(s)\right|_{s=0}>1$.

Lemma 4.2. For all $m \geq 1$,

$$
\left.\frac{d}{d s} \mu_{m}(s)\right|_{s=0}=\lim _{\epsilon \downarrow 0} \epsilon^{-1} \mu_{m}(\epsilon)=m R_{*}^{(m)} / \mathbb{E}[H]
$$

and hence,

$$
\left.\frac{d}{d s} \mu(s)\right|_{s=0}=\lim _{\epsilon \downarrow 0} \epsilon^{-1} \mu(\epsilon)=R_{*} .
$$

Proof. For $m \geq 1$ and $1 \leq k \leq m$,

$$
\pi_{m}^{k}(\epsilon)=\pi_{m}^{0}(\epsilon) \prod_{i=1}^{k} \frac{(m+1-i)\left(\beta_{G} \epsilon+(i-1) \beta_{L}\right)}{i \gamma} .
$$

Thus $\pi_{m}^{1}(\epsilon)=\frac{m \beta_{G}}{\gamma} \epsilon \pi_{m}^{0}(\epsilon)$ and for $2 \leq k \leq m$,

$$
\pi_{m}^{k}(\epsilon)=\frac{m \beta_{G}}{\gamma} \epsilon \pi_{m}^{0}(\epsilon) \prod_{i=2}^{k} \frac{(m+1-i)(i-1) \beta_{L}}{i \gamma}+o(\epsilon)
$$


with $\pi_{m}^{0}(\epsilon)=1-O(\epsilon)$. Therefore for $1 \leq k \leq m$,

$$
\pi_{m}^{k}(\epsilon)=\frac{m \beta_{G}}{\gamma} \epsilon\left(\frac{(m-1) !}{k(m-k) !}\right) \rho^{-(k-1)}+o(\epsilon) .
$$

Thus

$$
\begin{aligned}
\mu_{m}(\epsilon) & =\sum_{k=0}^{m} k \pi_{m}^{k}(\epsilon) \\
& =\left(\frac{m \beta_{G}}{\gamma} \epsilon\right) \sum_{k=1}^{m} k\left(\frac{(m-1) !}{k(m-k) !}\right) \rho^{-(k-1)}+o(\epsilon) \\
& =\frac{m}{\mathbb{E}[H]} R_{*}^{(m)} \epsilon+o(\epsilon)
\end{aligned}
$$

and so, $\lim _{\epsilon \downarrow 0} \epsilon^{-1} \mu_{m}(\epsilon)=m R_{*}^{(m)} / \mathbb{E}[H]$ as required.

The proceeding results are drawn together in the Lemma 4.3.

Lemma 4.3. For $R_{*} \leq 1$, the only $s \geq 0$ such that $\mu(s)=s$ is $s=0$.

For $R_{*}>1$, there exists a unique $s^{*}>0$ such that $\mu\left(s^{*}\right)=s^{*}$.

Moreover, for $s_{1}<s^{*}<s_{2}, s_{1}<\mu\left(s_{1}\right)$ and $\mu\left(s_{2}\right)<s_{2}$.

Before returning to $x(\cdot)$, we state two very useful general results for timeinhomogeneous Markov birth-death processes.

Consider any two continuous time, time-inhomogeneous Markov birth-death processes, labelled 1 and 2, with at time $u \geq 0$ transition matrices $H_{1}(u)$ and $H_{2}(u)$, respectively. For $u \geq 0$, let $X_{1}(u)$ and $X_{2}(u)$ denote the total number of individuals alive in processes 1 and 2 , respectively, at time $u$. Suppose that the maximum population size in both processes is $m \geq 1$, and that for $j=1,2$, the transition matrices $H_{j}(u)$ are $(m+1) \times(m+1)$ matrices of the form:

$$
\begin{array}{ll}
h_{i(i+1)}^{j}(u)>0 & 0 \leq i \leq m-1 \\
h_{i(i-1)}^{j}(u)=i \gamma & 1 \leq i \leq m \\
h_{i k}^{j}(u)=0 & |i-k| \geq 2 \\
h_{i i}^{j}(u)=-\sum_{k \neq i} h_{i k}^{j}(u) & 0 \leq i \leq m .
\end{array}
$$

Proposition 4.1. Suppose that for all $u \geq 0$ and $0 \leq i \leq m-1$,

$$
h_{i(i+1)}^{1}(u) \leq h_{i(i+1)}^{2}(u)
$$


and $X_{1}(0) \leq X_{2}(0)$. Then a coupling exists such that for all $t \geq 0$,

$$
X_{1}(t) \leq X_{2}(t) \quad \text { a.s. }
$$

For $j=1,2$ and $0 \leq s \leq t$, let $R_{j}(s, t)=\exp \left(\int_{s}^{t} H_{j}(u) d s\right)$. Then the Lemma 4.4 follows immediately from Proposition 4.1 by taking expectations.

Lemma 4.4. Suppose that for all $u \geq 0$ and $0 \leq i \leq m-1$,

$$
h_{i(i+1)}^{1}(u) \leq h_{i(i+1)}^{2}(u)
$$

and for $\mathbf{x}, \mathbf{y} \in \mathbb{R}^{m+1}$ with $\mathbf{x} \leq \mathbf{y}$. Then for all $0 \leq a \leq b$,

$$
\mathbf{x} R_{1}(a, b) \leq \mathbf{y} R_{2}(a, b)
$$

Corollary 4.2. Suppose that for all $m \geq 1, \mathbf{a}_{m}, \mathbf{b}_{m} \in \mathbb{R}^{m+1}, \mathbf{a}_{m} \leq \mathbf{b}_{m}$. Let $x_{\mathbf{a}}(0)=$ $\sum_{k=1}^{\infty} \omega_{k} \mathbf{k a}_{k}^{T}$ and $x_{\mathbf{b}}(0)=\sum_{k=1}^{\infty} \omega_{k} \mathbf{k} \mathbf{b}_{k}^{T}$ with

$$
x_{\mathbf{a}}(t)=\sum_{k=1}^{\infty} \omega_{k} \mathbf{k}\left\{\mathbf{a}_{k} S_{k}\left(0, t ; \mathbf{x}_{\mathbf{a}}\right)\right\}^{T}
$$

and

$$
x_{\mathbf{b}}(t)=\sum_{k=1}^{\infty} \omega_{k} \mathbf{k}\left\{\mathbf{b}_{k} S_{k}\left(0, t ; \mathbf{x}_{\mathbf{b}}\right)\right\}^{T} .
$$

Then for all $t \geq 0, x_{\mathbf{a}}(t) \leq x_{\mathbf{b}}(t)$ and for all $m \geq 1$,

$$
\mathbf{a}_{m} S_{m}\left(0, t ; \mathbf{x}_{\mathbf{a}}\right) \leq \mathbf{b}_{m} S_{m}\left(0, t ; \mathbf{x}_{\mathbf{b}}\right),
$$

and for $\mathbf{c}_{m} \leq \mathbf{d}_{m}$

$$
\mathbf{c}_{m} S_{m}\left(0, t ; \mathbf{x}_{\mathbf{a}}\right) \leq \mathbf{d}_{m} S_{m}\left(0, t ; \mathbf{x}_{\mathbf{a}}\right) .
$$

Proof. The Corollary follows by applying Lemma 4.4 to the households model.

Thus we have shown that the SIS household epidemic model satisfies a monotonicity condition. That is, two populations with identical household structure and disease dynamics can be coupled household by household such that if, for all $i \geq 1$, there are initially at least as many infectives in household $i$ in population 2 as in population 1 , then for all $t \geq 0$, there are at least as many infectives in household $i$ in population 2 as in population 1 , at time $t$.

We now consider the stationary distributions $\boldsymbol{\pi}_{m}(s)(m \geq 1, s \geq 0)$. 
Lemma 4.5. For $m \geq 1$ and $0 \leq r<s$, for all $t>0$,

$$
\boldsymbol{\pi}_{m}(r)<\boldsymbol{\pi}_{m}(r) \tilde{S}_{m}(t ; s)<\boldsymbol{\pi}_{m}(s)
$$

and

$$
\boldsymbol{\pi}_{m}(r)<\boldsymbol{\pi}_{m}(s) \tilde{S}_{m}(t ; r)<\boldsymbol{\pi}_{m}(s) .
$$

Lemmas 4.3 and 4.5 and Corollary 4.2 lead to the following key result.

Lemma 4.6. Let $R_{*}>1$ and let $s^{*}>0$, solve $\mu\left(s^{*}\right)=s^{*}$.

(i) For $s>s^{*}$, let $\mu(s)=s_{0}$. Then $s^{*}<s_{0}<s$, and for any $s_{0}<s_{1}<s$, there exists $t_{1}>0$ such that, for all $m \geq 1$,

$$
\boldsymbol{\pi}_{m}(s) S_{m}\left(0, t_{1} ; \mathbf{y}^{s}\right)<\boldsymbol{\pi}_{m}\left(s_{1}\right)
$$

where for $t, u \geq 0, y^{u}(t)=\sum_{k=1}^{\infty} \omega_{k} \mathbf{k}\left\{\boldsymbol{\pi}_{k}(u) S_{k}\left(0, t ; \mathbf{y}^{u}\right)\right\}^{T}$

(ii) For $0<r<s^{*}$, let $\mu(r)=r_{0}$. Then $r<r_{0}<s^{*}$ and for any $r<r_{1}<r_{0}$, there exists $t_{2}>0$ such that, for all $m \geq 1$,

$$
\boldsymbol{\pi}_{m}(r) S_{m}\left(0, t_{2} ; \mathbf{y}^{r}\right)>\boldsymbol{\pi}_{m}\left(r_{1}\right) .
$$

Proof. We shall prove statement $(i)$ with statement $(i i)$ following similarly.

Fix $s>s^{*}$ and $\mu(s)=s_{0}<s_{1}<s$.

Firstly, note that $y^{s}(0)=s_{0}$. Now suppose that there exists $t_{2}>0$ such that $y^{s}\left(t_{2}\right)>s_{0}$ and for all $0 \leq t<t_{2}, y^{s}(t) \leq s_{0}$. This is a contradiction since by Corollary 4.2

$$
\begin{aligned}
y^{s}\left(t_{2}\right) & =\sum_{k=1}^{\infty} \omega_{k} \mathbf{k}\left\{\boldsymbol{\pi}_{k}(s) S_{k}\left(0, t_{2} ; \mathbf{y}^{s}\right)\right\}^{T} \\
& \leq \sum_{k=1}^{\infty} \omega_{k} \mathbf{k}\left\{\boldsymbol{\pi}_{k}(s) \tilde{S}_{k}\left(t_{2} ; s_{0}\right)\right\}^{T} \\
& \leq \sum_{k=1}^{\infty} \omega_{k} \mathbf{k} \boldsymbol{\pi}_{k}(s)^{T}=s_{0}
\end{aligned}
$$

Thus for any $t_{2}>0$, for $y^{s}\left(t_{2}\right)>s_{0}$, we require that there exists $0<t_{3}<t_{2}$ such that $y^{s}\left(t_{3}\right)>s_{0}$. Or equivalently, there exists $t_{2}>0$, such that $y^{s}\left(t_{2}\right)>s_{0}$, only if

$$
\left.\frac{d}{d t} y^{s}(t)\right|_{t=0} \geq 0
$$


However,

$$
\begin{aligned}
\left.\frac{d}{d t} y^{s}(t)\right|_{t=0} & =\sum_{k=1}^{\infty} \omega_{k} \mathbf{k}\left\{\boldsymbol{\pi}_{k}(s) G_{k}\left(s_{0}\right)\right\}^{T} \\
& <\sum_{k=1}^{\infty} \omega_{k} \mathbf{k}\left\{\boldsymbol{\pi}_{k}(s) G_{k}(s)\right\}^{T}=\sum_{k=1}^{\infty} \omega_{k} \mathbf{k} \mathbf{0}^{T}=0 .
\end{aligned}
$$

Therefore for all $t \geq 0, y^{s}(t) \leq s_{0}$, and hence, by Corollary 4.2 , for all $m \geq 1$,

$$
\boldsymbol{\pi}_{m}(s) S_{m}\left(0, t ; \mathbf{y}^{s}\right) \leq \boldsymbol{\pi}_{m}(s) \tilde{S}_{m}\left(t ; s_{0}\right)
$$

Then since

$$
\boldsymbol{\pi}_{m}(s) \tilde{S}_{m}\left(t ; s_{0}\right) \rightarrow \boldsymbol{\pi}_{m}\left(s_{0}\right) \quad \text { as } t \rightarrow \infty
$$

it follows that there exists $t_{1}>0$ such that

$$
\boldsymbol{\pi}_{m}(s) \tilde{S}_{m}\left(t_{1} ; s_{0}\right)<\boldsymbol{\pi}_{m}\left(s_{1}\right)
$$

Hence statement $(i)$ follows by (4.3) and (4.4).

Lemma 4.7. Let $R_{*}>1$ and $s^{*}>0$ solve $\mu\left(s^{*}\right)=s^{*}$. Suppose that there exists $\epsilon>0$ such that for all $m \geq 1$ with $\omega_{m}>0, \boldsymbol{\zeta}_{m} \geq \boldsymbol{\pi}_{m}(\epsilon)$. Then

$$
x(t) \rightarrow s^{*} \quad \text { as } t \rightarrow \infty
$$

Proof. Fix $0<\epsilon<s^{*}$ such that for all $m \geq 1$,

$$
\mathbf{a}_{m}=\boldsymbol{\pi}_{m}(\epsilon) \leq \boldsymbol{\zeta}_{m} \leq \boldsymbol{\pi}_{m}(\infty)=\mathbf{b}_{m}
$$

For $t \geq 0$, let

$$
\begin{aligned}
& x_{\mathbf{a}}(t)=\sum_{k=1}^{\infty} \omega_{k} \mathbf{k}\left\{\mathbf{a}_{k} S_{k}\left(0, t ; \mathbf{x}_{\mathbf{a}}\right)\right\}^{T} \\
& x_{\mathbf{b}}(t)=\sum_{k=1}^{\infty} \omega_{k} \mathbf{k}\left\{\mathbf{b}_{k} S_{k}\left(0, t ; \mathbf{x}_{\mathbf{b}}\right)\right\}^{T}
\end{aligned}
$$

By successive applications of Lemma 4.6, for any $r_{1}<s^{*}<r_{2}$, there exists $t_{1}>0$ such that, for all $m \geq 1$,

$$
\begin{aligned}
& \boldsymbol{\pi}_{m}\left(r_{1}\right)<\mathbf{a}_{m} S_{m}\left(0, t_{1} ; \mathbf{x}_{\mathbf{a}}\right) \\
& \boldsymbol{\pi}_{m}\left(r_{2}\right)>\mathbf{b}_{m} S_{m}\left(0, t_{1} ; \mathbf{x}_{\mathbf{b}}\right)
\end{aligned}
$$

Moreover, Lemmas 4.5 and 4.6, can then be utilised to show that for all $t \geq 0$,

$$
\begin{aligned}
& \boldsymbol{\pi}_{m}\left(r_{1}\right)<\boldsymbol{\pi}_{m}\left(r_{1}\right) \tilde{S}_{m}\left(t ; \mu\left(r_{1}\right)\right)<\mathbf{a}_{m} S_{m}\left(0, t_{1}+t ; \mathbf{x}_{\mathbf{a}}\right) \\
& \boldsymbol{\pi}_{m}\left(r_{2}\right)>\boldsymbol{\pi}_{m}\left(r_{2}\right) \tilde{S}_{m}\left(t ; \mu\left(r_{2}\right)\right)>\mathbf{b}_{m} S_{m}\left(0, t_{1}+t ; \mathbf{x}_{\mathbf{b}}\right)
\end{aligned}
$$


Thus for all $t \geq t_{1}$,

$$
\begin{aligned}
& x_{\mathbf{a}}(t)>\sum_{k=1}^{\infty} \omega_{k} \mathbf{k} \boldsymbol{\pi}_{k}\left(r_{1}\right)^{T}=\mu\left(r_{1}\right) \\
& x_{\mathbf{b}}(t)<\sum_{k=1}^{\infty} \omega_{k} \mathbf{k} \boldsymbol{\pi}_{k}\left(r_{2}\right)^{T}=\mu\left(r_{2}\right) .
\end{aligned}
$$

By Lemma 4.2, for all $t \geq 0, x_{\mathbf{a}}(t) \leq x(t) \leq x_{\mathbf{b}}(t)$. Hence for all $t>t_{1}$,

$$
\mu\left(r_{1}\right)<x(t)<\mu\left(r_{2}\right)
$$

Since the above result holds for all $r_{1}<s^{*}<r_{2}$ and $\mu\left(s^{*}\right)=s^{*}$, the Lemma follows.

The equivalent result for the case where $R_{*} \leq 1$ is given in Lemma 4.8.

Lemma 4.8. Suppose that $R_{*} \leq 1$. Then

$$
x(t) \rightarrow 0 \quad \text { as } t \rightarrow \infty .
$$

Proof. The proof is similar to the case $R_{*}>1$, and hence, the details are omitted.

Lemma 4.7 shows that if $R_{*}>1$, then for most initial configurations of infectives, the mean number of infectives per household converges to $s^{*}>0$ as $t \rightarrow \infty$, i.e. an endemic equilibrium exists. Moreover, in equilibrium the proportion of households of size $m$ with $i$ infectives is given by $\pi_{m}^{i}\left(s^{*}\right)$. However, there is still some work to be done to achieve our goal of showing that if $x(0)>0$ and $R_{*}>1$ then $x(t) \rightarrow s^{*}$ as $t \rightarrow \infty$. Suppose that, for any $x(0)>0$, there exists $t_{1}>0$ and $\epsilon>0$, such that, for all $m \geq 1$, with $\omega_{m}>0$,

$$
\boldsymbol{\pi}_{m}(\epsilon)<\boldsymbol{\zeta}_{m} S_{m}\left(0, t_{1} ; \mathbf{x}\right)
$$

We can then apply Lemma 4.7 to prove the required result. Thus we proceed by showing that $t_{1}>0$ and $\epsilon>0$ exist.

Lemma 4.9. Suppose that $x(0)>0$. Then for all $m \geq 1$ and $t>0$,

$$
\boldsymbol{\zeta}_{m} S_{m}(0, t ; \mathbf{x})>(1,0, \ldots, 0)
$$

Proof. Note that for any $m \geq 1$ and $t>0$, if $\boldsymbol{\zeta}_{m}>(1,0, \ldots, 0)$ then

$$
\boldsymbol{\zeta}_{m} \tilde{S}_{m}(t ; 0)>(1,0, \ldots, 0)
$$


Therefore, by Lemma 4.2 , for all $t>0$,

$$
\begin{aligned}
x(t) & =\sum_{k=1}^{\infty} \omega_{k} \mathbf{k}\left\{\boldsymbol{\zeta}_{k} S_{k}(0, t ; \mathbf{x})\right\}^{T} \\
& \geq \sum_{k=1}^{\infty} \omega_{k} \mathbf{k}\left\{\boldsymbol{\zeta}_{k} \tilde{S}_{k}(t ; 0)\right\}^{T}>0 .
\end{aligned}
$$

Thus for any $t>0, \int_{0}^{t} x(s) d s>0$ corresponding to all individuals being subjected to total global infectious pressure $\beta_{G} \int_{0}^{t} x(s) d s>0$ up to time $t$. Hence for all $t>0$, each individual has a positive probability of being infectious at time $t$, and so, the Lemma follows.

Lemma 4.10. Suppose that $x(0)>0$. Then for all $m \geq 1$ and $t>0$, there exists $\epsilon_{m}>0$ such that

$$
\boldsymbol{\zeta}_{m} S_{m}(0, t ; \mathbf{x})>\boldsymbol{\pi}_{m}\left(\epsilon_{m}\right)
$$

Proof. Fix $m \geq 1$ and $t_{1}, t_{2}>0$ with $t=t_{1}+t_{2}$.

Note that by construction, for all $i \geq 1$ and $t_{2}>0, \tilde{s}_{i m}^{m}\left(t_{2} ; 0\right)>0$, where $\tilde{s}_{j k}^{m}\left(t_{2} ; s\right)$ $(0 \leq j, k \leq m)$ denotes a typical element of $\tilde{S}_{m}\left(t_{2} ; s\right)$. Thus by Lemma 4.9 for all $m \geq 1$, there exists $\delta_{m}>0$, such that

$$
\begin{aligned}
\boldsymbol{\zeta}_{m} S_{m}\left(0, t_{1}+t_{2} ; \mathbf{x}\right) & \geq \boldsymbol{\zeta}_{m} S_{m}\left(0, t_{1} ; \mathbf{x}\right) \tilde{S}_{m}\left(t_{2} ; 0\right) \\
& >\left(1-\delta_{m}, 0, \ldots, 0, \delta_{m}\right)
\end{aligned}
$$

Hence by taking $\epsilon_{m}>0$ such that $\pi_{m}^{0}\left(\epsilon_{m}\right)=1-\delta_{m}$, the Lemma is proved.

Theorem 4.1. Let $R_{*}>1$ and $s^{*}>0$ solve $\mu\left(s^{*}\right)=s^{*}$. Suppose that $x(0)=$ $\sum_{k=1}^{\infty} \omega_{k} \mathbf{k} \boldsymbol{\zeta}_{k}^{T}>0$. Then

$$
x(t) \rightarrow s^{*} \quad \text { as } t \rightarrow \infty .
$$

Proof. Suppose that there exists $M \geq 1$ such that for all $k>M, \omega_{k}=0$. Then it follows immediately from Lemmas 4.7 and 4.10 , that for $x(0)>0$,

$$
x(t) \rightarrow s^{*} \quad \text { as } t \rightarrow \infty .
$$

For $M \geq 1$, we can construct lower and upper bound approximations to the epidemic process where, for all $t \geq 0$, all individuals in households of size $k>M$ are susceptible and infectious, respectively. Since we can take $M$ to be arbitrarily large and 
$\sum_{k=M+1}^{\infty} k \omega_{k} \rightarrow 0$ as $M \rightarrow \infty$, we can make the lower and upper bound approximations to the epidemic process arbitrarily close to the actual epidemic process. The Theorem then follows.

\section{Fluctuations and Gaussian limit processes}

In this section, we aim to extend the results of Section 3 to obtain a Gaussian limit process for the fluctuations in the mean number of infectives per household $X_{n}(\cdot)$ about the deterministic limit $x(\cdot)$. In other words, for any $T>0$, we wish to show that $V_{n}(\cdot)=\sqrt{n}\left(X_{n}(\cdot)-x(\cdot)\right)$ converges to a Gaussian process $V(\cdot)$ on $[0, T]$. This is possible for $Q \sim \operatorname{Exp}(\gamma)$ since we have an explicit expression for $x(\cdot)$, see Section 4 . For more general $Q$, we are unable to obtain a Gaussian limit but are able to show that, for any $T \geq 0,\left\{\sup _{0 \leq s \leq T}\left|V_{n}(s)\right| ; n \geq 1\right\}$ is stochastically bounded.

Firstly, for $n \geq 1, t \geq 0$ and $\mathbf{y}=\{y(s) ; s \geq 0\}$, let

$$
Y_{n}(t ; \mathbf{y})=\frac{1}{\sqrt{n}} \sum_{i=1}^{n}\left\{\theta_{i}(t ; \mathbf{y})-\mathbb{E}\left[\theta_{i}(t ; \mathbf{y})\right]\right\}
$$

Note that, for $s \geq 0$,

$$
V_{n}(s)=Y_{n}\left(s ; \mathbf{X}_{n}\right)+\frac{1}{\sqrt{n}} \sum_{i=1}^{n}\left\{\mathbb{E}\left[\theta_{i}\left(s ; \mathbf{X}_{n}\right) \mid \mathbf{X}_{n}\right]-\mathbb{E}\left[\theta_{i}(s ; \mathbf{x})\right]\right\}
$$

Thus we begin by analysing $Y_{n}\left(\cdot ; \mathbf{X}_{n}\right)$, and we shall assume that $\mathbb{E}\left[H^{4}\right]<\infty$.

For $m \in \mathbb{N}$ and $\mathbf{t}=\left(t_{1}, t_{2}, \ldots, t_{m}\right), \gamma=\left(\gamma_{1}, \gamma_{2}, \ldots, \gamma_{m}\right) \in \mathbb{R}^{m}$, let

$$
\begin{aligned}
Z_{n}^{\gamma}(\mathbf{t} ; \mathbf{y}) & =\sum_{k=1}^{m} \gamma_{k} Y_{n}\left(t_{k} ; \mathbf{y}\right) \\
& =\frac{1}{\sqrt{n}} \sum_{i=1}^{n}\left\{\sum_{k=1}^{m} \gamma_{k}\left(\theta_{i}\left(t_{k} ; \mathbf{y}\right)-\mathbb{E}\left[\theta_{i}\left(t_{k} ; \mathbf{y}\right)\right]\right)\right\},
\end{aligned}
$$

and

$$
z_{n}^{\gamma}(\mathbf{t} ; \mathbf{y})=\frac{1}{n} \sum_{i=1}^{n}\left\{\sum_{j=1}^{m} \sum_{k=1}^{m} \gamma_{j} \gamma_{k} \operatorname{cov}\left(\theta_{i}\left(t_{j} ; \mathbf{y}\right), \theta_{i}\left(t_{k} ; \mathbf{y}\right)\right)\right\} .
$$

Lemma 5.1. For any $m \in \mathbb{N}$ and $\mathbf{t} \in \mathbb{R}^{m}$, with $\mathbf{t} \geq 0$,

$$
\left(Y_{n}\left(t_{1} ; \mathbf{x}\right), Y_{n}\left(t_{2} ; \mathbf{x}\right), \ldots, Y_{n}\left(t_{m} ; \mathbf{x}\right)\right) \stackrel{D}{\longrightarrow}\left(Y\left(t_{1} ; \mathbf{x}\right), Y\left(t_{2} ; \mathbf{x}\right), \ldots, Y\left(t_{m} ; \mathbf{x}\right)\right) \quad \text { as } n \rightarrow \infty
$$


where $\mathbf{Y}$ is a multivariate normal distribution with mean $\mathbf{0}$ and covariance matrix $\Sigma(\mathbf{t})$, where the $(j, k)^{\text {th }}$ element of $\Sigma(\mathbf{t})$ is

$$
\sum_{i=1}^{\infty} \omega_{i} \sum_{l=0}^{i} \zeta_{i l} \operatorname{cov}\left(\chi_{i l}\left(t_{j} ; \mathbf{x}\right), \chi_{i l}\left(t_{k} ; \mathbf{x}\right)\right)
$$

Proof. Fix $\gamma \in \mathbb{R}^{m}$ and then since $\mathbb{E}\left[H^{2}\right]<\infty$, it follows by Lindeberg's Central Limit Theorem (see, for example, [6], Theorem 7.2) that

$$
Z_{n}^{\gamma}(\mathbf{t} ; \mathbf{x}) / z_{n}^{\gamma}(\mathbf{t} ; \mathbf{x}) \stackrel{D}{\longrightarrow} N(0,1) \quad \text { as } n \rightarrow \infty .
$$

Note that

$$
\begin{aligned}
z_{n}^{\gamma}(\mathbf{t} ; \mathbf{x}) & =\sum_{i=1}^{\infty} \omega_{i}^{n} \sum_{l=0}^{i} \zeta_{i l}^{n} \sum_{j=1}^{m} \sum_{k=1}^{m} \gamma_{j} \gamma_{k} \operatorname{cov}\left(\chi_{i l}\left(t_{j} ; \mathbf{x}\right), \chi_{i l}\left(t_{k} ; \mathbf{x}\right)\right) \\
& \rightarrow \sum_{j=1}^{m} \sum_{k=1}^{m} \gamma_{j} \gamma_{k} \sum_{i=1}^{\infty} \omega_{i} \sum_{l=0}^{i} \zeta_{i l} \operatorname{cov}\left(\chi_{i l}\left(t_{j} ; \mathbf{x}\right), \chi_{i l}\left(t_{k} ; \mathbf{x}\right)\right) \\
& =z^{\gamma}(\mathbf{t} ; \mathbf{x}), \quad \text { say } \quad \text { as } n \rightarrow \infty
\end{aligned}
$$

Therefore $Z_{n}^{\gamma}(\mathbf{t} ; \mathbf{x}) \stackrel{D}{\longrightarrow} N\left(0, z^{\gamma}(\mathbf{t} ; \mathbf{x})\right)$ as $n \rightarrow \infty$ and the Lemma follows by the Cramér-Wold device (see, for example, [6], pages 48-49).

In order to make use of Lemma 5.1, we need to show that $\left\{Y_{n}\left(t ; \mathbf{X}_{n}\right)\right\}$ has the same asymptotic limiting distribution as $\left\{Y_{n}(t ; \mathbf{x})\right\}$ as $n \rightarrow \infty$.

Lemma 5.2. For any $t \geq 0$ and $\epsilon>0$,

$$
\mathbb{P}\left(\left|Y_{n}\left(t ; \mathbf{X}_{n}\right)-Y_{n}(t ; \mathbf{x})\right|>\epsilon\right) \rightarrow 0 \quad \text { as } n \rightarrow \infty
$$

Proof. As noted in [14], Lemma 5.4, by Chebychev's inequality, for any $\epsilon>0$,

$$
\begin{aligned}
\mathbb{P}\left(\left|Y_{n}\left(t ; \mathbf{X}_{n}\right)-Y_{n}(t ; \mathbf{x})\right|>\epsilon\right) & \leq \frac{1}{\epsilon^{2}} \mathbb{E}\left[\operatorname{var}\left(Y_{n}\left(t ; \mathbf{X}_{n}\right)-Y_{n}(t ; \mathbf{x}) \mid \mathbf{X}_{n}\right)\right] \\
& =\frac{1}{\epsilon^{2}} \frac{1}{n} \sum_{i=1}^{n} \mathbb{E}\left[\operatorname{var}\left(\theta_{i}^{n}\left(t ; \mathbf{X}_{n}\right)-\theta_{i}^{n}(t ; \mathbf{x}) \mid \mathbf{X}_{n}\right)\right] \\
& \leq \frac{1}{\epsilon^{2}} \frac{1}{n} \sum_{i=1}^{n} h_{i}^{2} \mathbb{E}\left[\mathbb{P}\left(\theta_{i}^{n}\left(t ; \mathbf{X}_{n}\right) \neq \theta_{i}^{n}(t ; \mathbf{x}) \mid \mathbf{X}_{n}\right)\right]
\end{aligned}
$$

The righthand side of (5.1) is equal to

$$
\frac{1}{\epsilon^{2}} \sum_{k=1}^{\infty} k^{2} \omega_{k} \sum_{l=0}^{k} \zeta_{k l}^{n} \mathbb{E}\left[\mathbb{P}\left(\chi_{k l}\left(t ; \mathbf{X}_{n}\right) \neq \chi_{k l}(t ; \mathbf{x}) \mid \mathbf{X}_{n}\right)\right]
$$


By similar arguments to those employed in Lemma 3.1, for $k \geq 1$ and $0 \leq l \leq k$,

$$
\begin{aligned}
\mathbb{P}\left(\chi_{k l}\left(t ; \mathbf{X}_{n}\right) \neq \chi_{k l}(t ; \mathbf{x}) \mid \mathbf{X}_{n}\right) & \leq k \beta_{G} \int_{0}^{t}\left|X_{n}(s)-x(s)\right| d s \\
& \leq k \beta_{G} t \sup _{0 \leq s \leq t}\left|X_{n}(s)-x(s)\right|
\end{aligned}
$$

Thus it follows from (5.1) that

$$
\mathbb{P}\left(\left|Y_{n}\left(t ; \mathbf{X}_{n}\right)-Y_{n}(t ; \mathbf{x})\right|>\epsilon\right) \leq \frac{1}{\epsilon^{2}} \beta_{G} t \mathbb{E}\left[\sup _{0 \leq s \leq t}\left|X_{n}(s)-x(s)\right|\right] \sum_{k=1}^{\infty} k^{3} \omega_{k}^{n} .
$$

Note that by Lemma 3.2, for all $t \geq 0, \sup _{0 \leq s \leq t}\left|X_{n}(s)-x(s)\right| \stackrel{p}{\longrightarrow} 0$ as $n \rightarrow \infty$, and then since for all $s \geq 0, X_{n}(s) \leq \sum_{k=1}^{\infty} k \omega_{k}^{n}$, it follows that

$$
\mathbb{E}\left[\sup _{0 \leq s \leq t}\left|X_{n}(s)-x(s)\right|\right] \rightarrow 0 \quad \text { as } n \rightarrow \infty
$$

Therefore the righthand side of (5.2) converges to 0 as $n \rightarrow \infty$ and the Lemma is proved.

Corollary 5.1. For any $m \in \mathbb{N}$ and $\mathbf{t} \in \mathbb{R}^{m}$ with $\mathbf{t} \geq \mathbf{0}$,

$$
\left(Y_{n}\left(t_{1} ; \mathbf{X}_{n}\right), Y_{n}\left(t_{2} ; \mathbf{X}_{n}\right), \ldots, Y_{n}\left(t_{m} ; \mathbf{X}_{n}\right)\right) \stackrel{D}{\longrightarrow}\left(Y\left(t_{1} ; \mathbf{x}\right), Y\left(t_{2} ; \mathbf{x}\right), \ldots, Y\left(t_{m} ; \mathbf{x}\right)\right)
$$

as $n \rightarrow \infty$, where $Y(\cdot ; \mathbf{x})$ is defined in Lemma 5.1.

Proof. The Corollary follows immediately from Lemmas 5.1 and 5.2 by utilising [6], Theorem 4.1.

Therefore we have established that the finite dimensional distributions of $Y_{n}\left(\cdot ; \mathbf{X}_{n}\right)$ converges to the finite dimensional distributions of $Y(\cdot ; \mathbf{x})$, a Gaussian process. The next step is to show that $\left\{Y_{n}\left(\cdot ; \mathbf{X}_{n}\right)\right\}$ is tight. Hence, for $T>0$, by defining $Y(\cdot ; \mathbf{x})$ and $Y_{n}\left(\cdot ; \mathbf{X}_{n}\right)(n \geq 1)$ on $D[0, T]$ endowed with the Skorohod topology, we will be able to show that $Y_{n}\left(\cdot ; \mathbf{X}_{n}\right) \Rightarrow Y(\cdot ; \mathbf{x})$ on $D[0, T]$.

Lemma 5.3. For any $\mathbf{y}=\{y(s) ; s \geq 0\}$ and $L>0$ such that $\sup _{s \geq 0}|y(s)| \leq L$ and $0 \leq s \leq t \leq u$, there exists a finite constant, $D_{L}$, independent of $s, t$ and $u$, such that for all $n \geq 1$,

$$
\mathbb{E}\left[\left(Y_{n}(t ; \mathbf{y})-Y_{n}(s ; \mathbf{y})\right)^{2}\left(Y_{n}(u ; \mathbf{y})-Y_{n}(t ; \mathbf{y})\right)^{2}\right] \leq D_{L}(u-s)^{2}
$$


Proof. For $n \geq 1$ and $1 \leq i \leq n$, let $\tilde{\theta}_{i}^{n}(t ; \mathbf{y})=\theta_{i}^{n}(t ; \mathbf{y})-\mathbb{E}\left[\theta_{i}^{n}(t ; \mathbf{y})\right]$. Since the distinct households are conditionally independent given $\mathbf{y}$ and $\mathbb{E}\left[\tilde{\theta}_{i}^{n}(t ; \mathbf{y})\right]=0$, we have that

$$
\begin{aligned}
\mathbb{E} & {\left[\left(Y_{n}(t ; \mathbf{y})-Y_{n}(s ; \mathbf{y})\right)^{2}\left(Y_{n}(u ; \mathbf{y})-Y_{n}(t ; \mathbf{y})\right)^{2}\right] } \\
\leq & \frac{1}{n^{2}} \sum_{i=1}^{n} \sum_{j=1}^{n} \sum_{k=1}^{n} \sum_{l=1}^{n} \mathbb{E}\left[\left\{\tilde{\theta}_{i}^{n}(t ; \mathbf{y})-\tilde{\theta}_{i}^{n}(s ; \mathbf{y})\right\}\left\{\tilde{\theta}_{j}^{n}(t ; \mathbf{y})-\tilde{\theta}_{j}^{n}(s ; \mathbf{y})\right\}\right. \\
& \left.\times\left\{\tilde{\theta}_{k}^{n}(u ; \mathbf{y})-\tilde{\theta}_{k}^{n}(t ; \mathbf{y})\right\}\left\{\tilde{\theta}_{l}^{n}(u ; \mathbf{y})-\tilde{\theta}_{l}^{n}(u ; \mathbf{y})\right\}\right] \\
\leq & \frac{1}{n^{2}}\left\{\sum_{i=1}^{n} \mathbb{E}\left[\left(\tilde{\theta}_{i}^{n}(t ; \mathbf{y})-\tilde{\theta}_{i}^{n}(s ; \mathbf{y})\right)^{2}\right]\right\}\left\{\sum_{j=1}^{n} \mathbb{E}\left[\left(\tilde{\theta}_{j}^{n}(u ; \mathbf{y})-\tilde{\theta}_{j}^{n}(t ; \mathbf{y})\right)^{2}\right]\right\} \\
+ & \frac{2}{n^{2}}\left\{\sum_{i=1}^{n} \mathbb{E}\left[\left(\tilde{\theta}_{i}^{n}(t ; \mathbf{y})-\tilde{\theta}_{i}^{n}(s ; \mathbf{y})\right)\left(\tilde{\theta}_{i}^{n}(u ; \mathbf{y})-\tilde{\theta}_{i}^{n}(t ; \mathbf{y})\right)\right]\right\}^{2} \\
+ & \frac{1}{n^{2}} \sum_{i=1}^{n} \mathbb{E}\left[\left(\tilde{\theta}_{i}^{n}(t ; \mathbf{y})-\tilde{\theta}_{i}^{n}(s ; \mathbf{y})\right)^{2}\left(\tilde{\theta}_{i}^{n}(u ; \mathbf{y})-\tilde{\theta}_{i}^{n}(t ; \mathbf{y})\right)^{2}\right] .
\end{aligned}
$$

Note that

$$
\begin{aligned}
\mathbb{E}\left[\left(\tilde{\theta}_{i}(t ; \mathbf{y})-\tilde{\theta}_{i}(s ; \mathbf{y})\right)^{2}\right] & =\operatorname{var}\left(\theta_{i}(t ; \mathbf{y})-\theta_{i}(s ; \mathbf{y})\right) \\
& \leq \mathbb{E}\left[\left\{\theta_{i}(t ; \mathbf{y})-\theta_{i}(s ; \mathbf{y})\right\}^{2}\right] \\
& \leq h_{i}^{2} \mathbb{P}\left(\theta_{i}(t ; \mathbf{y}) \neq \theta_{i}(s ; \mathbf{y})\right)
\end{aligned}
$$

Now $\mathbb{P}\left(\theta_{i}(t ; \mathbf{y}) \neq \theta_{i}(s ; \mathbf{y})\right)$ is less than the probability that there are no infections or removals within household $i$ in the time interval $(s, t]$.

Infections take place within household $i$ at the points of an inhomogeneous Poisson point process with rate $\left(h_{i}-\theta_{i}(s ; \mathbf{y})\right)\left\{\beta_{G} y(s)+\beta_{L} \theta_{i}(s ; \mathbf{y})\right\}$. Therefore the probability that there are no infections in household $i$ in the interval $(s, t]$ is greater than or equal to $\exp \left(-\frac{1}{4}\left\{4 \beta_{G} h_{i} L+\beta_{L} h_{i}^{2}\right\}(t-s)\right)$ since $\sup _{r \geq 0}|y(r)| \leq L$. A similar argument shows that the probability there are no removals within household $i$ in the interval $(s, t]$ is greater than or equal to $\{1-\alpha(t-s)\}^{h_{i}}$ where $\alpha=\sup _{x \geq 0} f_{Q}(x)$.

Thus it follows from (5.4) that

$$
\begin{aligned}
& \mathbb{E}\left[\left(\tilde{\theta}_{i}(t ; \mathbf{y})-\tilde{\theta}_{i}(s ; \mathbf{y})\right)^{2}\right] \\
\leq & h_{i}^{2}\left\{1-\exp \left(-\frac{1}{4}\left\{4 \beta_{G} h_{i} L+\beta_{L} h_{i}^{2}\right\}(t-s)\right)\right\}+h_{i}^{2}\left\{1-(1-\alpha(t-s))^{h_{i}}\right\} \\
\leq & h_{i}^{2}\left(\frac{1}{4}\left\{4 \beta_{G} h_{i} L+\beta_{L} h_{i}^{2}\right\}(t-s)\right)+h_{i}^{2}\left(h_{i} \alpha(t-s)\right) .
\end{aligned}
$$


Therefore there exists a constant $C_{L}<\infty$, independent of $t, s$ and $h_{i}$, such that

$$
\mathbb{E}\left[\left(\tilde{\theta}_{i}(t ; \mathbf{y})-\tilde{\theta}_{i}(s ; \mathbf{y})\right)^{2}\right] \leq C_{L} h_{i}^{4}(t-s) .
$$

Similarly, we can show that

$$
\left|\mathbb{E}\left[\left\{\tilde{\theta}_{i}(t ; \mathbf{y})-\tilde{\theta}_{i}(s ; \mathbf{y})\right\}\left\{\tilde{\theta}_{i}(u ; \mathbf{y})-\tilde{\theta}_{i}(t ; \mathbf{y})\right\}\right]\right| \leq 2 h_{i}^{4} C_{L}(u-s)
$$

and

$$
\mathbb{E}\left[\left\{\tilde{\theta}_{i}(t ; \mathbf{y})-\tilde{\theta}_{i}(s ; \mathbf{y})\right\}^{2}\left\{\tilde{\theta}_{i}(u ; \mathbf{y})-\tilde{\theta}_{i}(t ; \mathbf{y})\right\}^{2}\right] \leq 6 C_{L}^{2} h_{i}^{8}(u-s)^{2} .
$$

Therefore it follows from (5.3-5.7) that

$$
\begin{aligned}
& \mathbb{E}\left[\left(Y_{n}(t ; \mathbf{y})-Y_{n}(s ; \mathbf{y})\right)^{2}\left(Y_{n}(u ; \mathbf{y})-Y_{n}(t ; \mathbf{y})\right)^{2}\right] \\
\leq & \left\{\frac{1}{n} \sum_{i=1}^{n} C_{L} h_{i}^{4}(u-s)\right\}^{2}+2\left\{\frac{1}{n} \sum_{i=1}^{n} 2 C_{L} h_{i}^{4}(u-s)\right\}^{2} \\
& +\frac{1}{n^{2}} \sum_{i=1}^{n} 6 C_{L}^{2} h_{i}^{8}(u-s)^{2} \\
\leq & 11 C_{L}^{2}(u-s)^{2}\left\{\sum_{k=1}^{\infty} k^{4} \omega_{k}^{n}\right\}
\end{aligned}
$$

Thus if $D_{L}=11 C_{L}^{2}\left(\mathbb{E}\left[H^{4}\right]+1\right)$, then $0<D_{L}<\infty$ and there exists $n_{0} \in \mathbb{N}$ such that for all $n \geq n_{0}$,

$$
\mathbb{E}\left[\left(Y_{n}(t ; \mathbf{y})-Y_{n}(s ; \mathbf{y})\right)^{2}\left(Y_{n}(u ; \mathbf{y})-Y_{n}(t ; \mathbf{y})\right)^{2}\right] \leq D_{L}(u-s)^{2}
$$

as required.

The following Corollary is then an immediate consequence of Lemma 5.3.

Corollary 5.2. Let $A=\mathbb{E}[H]$. Then for any $0 \leq s \leq t \leq u$, there exists $n_{0} \in \mathbb{N}$ such that for all $n \geq n_{0}$,

$$
\mathbb{E}\left[\left(Y_{n}\left(t ; \mathbf{X}_{n}\right)-Y_{n}\left(s ; \mathbf{X}_{n}\right)\right)^{2}\left(Y_{n}\left(u ; \mathbf{X}_{n}\right)-Y_{n}\left(t ; \mathbf{X}_{n}\right)\right)^{2}\right] \leq D_{A}(u-s)^{2}
$$

Hence, for any $T>0$,

$$
Y_{n}\left(\cdot ; \mathbf{X}_{n}\right) \Rightarrow Y(\cdot ; \mathbf{x})
$$

on $D[0, T]$ as $n \rightarrow \infty$. 
Proof. For $r>0$, let $\mathcal{G}_{r}=\left\{\mathbf{y}=\{y(s) ; s \geq 0\}: \sup _{s \geq 0} y(s) \leq r\right\}$ and note that $\mathbf{X}_{n} \in \mathcal{G}_{A}$, almost surely.

Therefore by Lemma 5.3,

$$
\begin{aligned}
& \mathbb{E}\left[\left(Y_{n}\left(t ; \mathbf{X}_{n}\right)-Y_{n}\left(s ; \mathbf{X}_{n}\right)\right)^{2}\left(Y_{n}\left(u ; \mathbf{X}_{n}\right)-Y_{n}\left(t ; \mathbf{X}_{n}\right)\right)^{2}\right] \\
= & \mathbb{E}\left[\mathbb{E}\left[\left(Y_{n}\left(t ; \mathbf{X}_{n}\right)-Y_{n}\left(s ; \mathbf{X}_{n}\right)\right)^{2}\left(Y_{n}\left(u ; \mathbf{X}_{n}\right)-Y_{n}\left(t ; \mathbf{X}_{n}\right)\right)^{2} \mid \mathbf{X}_{n}\right]\right] \\
\leq & \sup _{\mathbf{y} \in \mathcal{G}_{A}} \mathbb{E}\left[\left(Y_{n}(t ; \mathbf{y})-Y_{n}(s ; \mathbf{y})\right)^{2}\left(Y_{n}(u ; \mathbf{y})-Y_{n}(t ; \mathbf{y})\right)^{2}\right] \\
\leq & D_{A}(u-s)^{2}
\end{aligned}
$$

Thus (5.8) is proved and by [6], Theorem 15.6 the Lemma follows immediately from Corollary 5.1 and (5.8).

The final step in the analysis of $\left\{Y_{n}\left(\cdot ; \mathbf{X}_{n}\right)\right\}$ before returning to $\left\{V_{n}(\cdot)\right\}$ is to show that the limiting Gaussian process $Y(\cdot ; \mathbf{x})$ has almost surely continuous sample paths.

Lemma 5.4. The Gaussian process $Y(\cdot ; \mathbf{x})$ has a continuous version (see, [15], page $59)$, and hence, for $T>0, Y(\cdot ; \mathbf{x})$ has almost surely continuous sample paths on $D[0, T]$.

Proof. Fix $T>0$. By similar arguments to those employed in Lemma 5.3, it is straightforward to show that, there exists, a constant $C<\infty$ such that for all $0 \leq$ $s, t \leq T$

$$
\begin{aligned}
& |\mathbb{E}[Y(s ; \mathbf{x}) Y(t ; \mathbf{x})]-\mathbb{E}[Y(t ; \mathbf{x}) Y(t ; \mathbf{x})]| \\
= & \left|\sum_{i=1}^{\infty} \omega_{i} \sum_{l=0}^{i} \zeta_{i l}\left\{\operatorname{cov}\left(\chi_{i l}(s ; \mathbf{x})-\chi_{i l}(t ; \mathbf{x}), \chi_{i l}(t ; \mathbf{x})\right)\right\}\right| \\
\leq & C|s-t|
\end{aligned}
$$

The Lemma then follows by [15], Chapter I, Corollary 25.6.

The following Theorem utilises Corollary 5.2 and Lemma 5.4 to show that for all $T>0, V_{n}$ is stochastically bounded on $[0, T]$. We shall then focus on the case $Q \sim$ $\operatorname{Exp}(\gamma)$ where an explicit Gaussian limit for $V_{n}$ can be obtained.

Theorem 5.1. For any $T>0,\left\{\sup _{0 \leq s \leq T} V_{n}(s) ; n \geq 1\right\}$ is stochastically bounded. 
Proof. Note that, for all $t \geq 0$

$$
V_{n}(t)=Y_{n}\left(t ; \mathbf{X}_{n}\right)+\sqrt{n}\left\{\frac{1}{n} \sum_{i=1}^{n}\left(\mathbb{E}\left[\theta_{i}\left(t ; \mathbf{X}_{n}\right) \mid \mathbf{X}_{n}\right]-\mathbb{E}\left[\theta_{i}(t ; \mathbf{x})\right]\right)\right\} .
$$

Now using identical arguments to those employed in Lemma 3.1,

$$
\mid \mathbb{E}\left[\left[\theta_{i}\left(t ; \mathbf{X}_{n}\right) \mid \mathbf{X}_{n}\right]-\mathbb{E}\left[\theta_{i}(t ; \mathbf{x})\right] \leq h_{i} \int_{0}^{t} h_{i} \beta_{G}\left|X_{n}(s)-x(s)\right| d s\right.
$$

Hence,

$$
\begin{aligned}
& \sqrt{n}\left\{\frac{1}{n} \sum_{i=1}^{n}\left(\mathbb{E}\left[\theta_{i}\left(t ; \mathbf{X}_{n}\right) \mid \mathbf{X}_{n}\right]-\mathbb{E}\left[\theta_{i}(t ; \mathbf{x})\right]\right)\right\} \\
\leq & \sqrt{n}\left\{\frac{1}{n} \sum_{i=1}^{n} h_{i}^{2} \beta_{G} \int_{0}^{t}\left|X_{n}(s)-x(s)\right| d s\right\} \\
= & \beta_{G}\left\{\sum_{k=1}^{\infty} k^{2} \omega_{k}^{n}\right\} \int_{0}^{t}\left|V_{n}(s)\right| d s
\end{aligned}
$$

Thus,

$$
\begin{aligned}
\sup _{0 \leq s \leq T}\left|V_{n}(s)\right| & \leq \sup _{0 \leq s \leq T}\left|Y_{n}\left(s ; \mathbf{X}_{n}\right)\right|+\beta_{G}\left\{\sum_{k=1}^{\infty} k^{2} \omega_{k}^{n}\right\} \sup _{0 \leq s \leq T} \int_{0}^{s}\left|V_{n}(u)\right| d u \\
& \leq \sup _{0 \leq s \leq t}\left|Y_{n}\left(s ; \mathbf{X}_{n}\right)\right|+\beta_{G}\left\{\sum_{k=1}^{\infty} k^{2} \omega_{k}^{n}\right\} \int_{0}^{T} \sup _{0 \leq u \leq s}\left|V_{n}(u)\right| d u
\end{aligned}
$$

and hence, by Gronwall's inequality and Corollary 5.2,

$$
\begin{aligned}
\sup _{0 \leq s \leq T}\left|V_{n}(s)\right| & \leq\left(\sup _{0 \leq s \leq T}\left|Y_{n}\left(s ; \mathbf{X}_{n}\right)\right|\right) \exp \left(T \beta_{G} \sum_{k=1}^{\infty} k^{2} \omega_{k}^{n}\right) \\
& \stackrel{D}{\longrightarrow}\left(\sup _{0 \leq s \leq T}|Y(s ; \mathbf{x})|\right) \exp \left(T \beta_{G} \sum_{k=1}^{\infty} k^{2} \omega_{k}^{n}\right) \quad \text { as } n \rightarrow \infty .
\end{aligned}
$$

The Theorem follows since $Y(\cdot ; \mathbf{x})$ is continuous (see, Lemma 5.4).

\subsection{Exponential Infectious periods}

For $k \geq 1$, let $B_{k}$ denote a $(k+1) \times(k+1)$ matrix with typical entries $b_{i j}^{k}(0 \leq i, j \leq k)$ where $b_{i(i+1)}^{k}=k-i, b_{i i}^{k}=-(k-i)(0 \leq i \leq k-1)$ and $b_{i j}^{k}=0$ otherwise. It then follows that

$$
\sqrt{n} \int_{0}^{t}\left\{G_{k}\left(X_{n}(s)\right)-G_{k}(x(s))\right\} d s=\beta_{G}\left\{\int_{0}^{t} V_{n}(s) d s\right\} B_{k} .
$$


We shall require that there exists $a>0$, such that $\mathbb{E}[\exp (a H)]<\infty$.

Note that

$$
\begin{aligned}
& \sqrt{n} \sum_{k=1}^{\infty} \omega_{k} \mathbf{k}\left(\left\{\boldsymbol{\zeta}_{k}^{n} S_{k}\left(0, t ; \mathbf{X}_{n}\right)\right\}^{T}-\left\{\boldsymbol{\zeta}_{k} S_{k}(0, t ; \mathbf{x})\right\}^{T}\right) \\
= & \sqrt{n} \sum_{k=1}^{\infty} \omega_{k}\left\{\left(\boldsymbol{\zeta}_{k}^{n}-\boldsymbol{\zeta}_{k}\right) S_{k}\left(0, t ; \mathbf{X}_{n}\right)\right\}^{T}+\sqrt{n} \sum_{k=1}^{\infty} \omega_{k}\left\{S_{k}\left(0, t ; \mathbf{X}_{n}\right)-S_{k}(0, t ; \mathbf{x})\right\}^{T} \boldsymbol{\zeta}_{k}^{T} \\
= & \epsilon_{n}^{1}(t)+\sqrt{n} \sum_{k=1}^{\infty} \omega_{k}\left\{S_{k}\left(0, t ; \mathbf{X}_{n}\right)-S_{k}(0, t ; \mathbf{x})\right\}^{T} \boldsymbol{\zeta}_{k}^{T}, \quad \text { say. }
\end{aligned}
$$

Therefore provided

$$
\sqrt{n} \sum_{k=1}^{\infty} \omega_{k} k \sum_{l=0}^{k}\left|\zeta_{k l}^{n}-\zeta_{k l}\right| \rightarrow 0 \quad \text { as } n \rightarrow \infty
$$

it will follow that for all $T \geq 0, \boldsymbol{\epsilon}_{n}^{1} \Rightarrow \mathbf{0}$ as $n \rightarrow \infty$ on $[0, T]$.

Lemma 5.5. Suppose that there exists $a>0$, such that $\mathbb{E}[\exp (a H)]<\infty$. For all $n \geq 1$ and $t \geq 0$, let

$$
\begin{aligned}
& \sqrt{n} \sum_{k=1}^{\infty} \omega_{k} \mathbf{k}\left\{S_{k}\left(0, t ; \mathbf{X}_{n}\right)^{T}-S_{k}(0, t ; \mathbf{x})^{T}\right\} \boldsymbol{\zeta}_{k}^{T} \\
= & \beta_{G} \int_{0}^{t} V_{n}(s) d s \sum_{k=1}^{\infty}\left\{\omega_{k} \mathbf{k} B_{k}^{T} S_{k}(0, t ; \mathbf{x})^{T} \boldsymbol{\zeta}_{k}^{T}\right\}+\epsilon_{n}^{2}(t),
\end{aligned}
$$

where for $k \geq 1$,

$$
C_{k}^{n}(t)=\exp \left(\frac{\beta_{G}}{\sqrt{n}} \int_{0}^{t} V_{n}(s) d s B_{k}\right)^{T}-I_{k+1}-\frac{\beta_{G}}{\sqrt{n}} \int_{0}^{t} V_{n}(s) d s B_{k}^{T}
$$

and

$$
\epsilon_{n}^{2}(t)=\sqrt{n} \sum_{k=1}^{\infty} \omega_{k} \mathbf{k} C_{k}^{n}(t) S_{k}(0, t ; \mathbf{x})^{T} \boldsymbol{\zeta}_{k}^{T}
$$

with $I_{k+1}$ denoting the $k+1$-dimensional identity matrix.

Then for all $T \geq 0, \boldsymbol{\epsilon}_{n}^{2} \Rightarrow \mathbf{0}$ as $n \rightarrow \infty$ on $[0, T]$.

Proof. For all $k \geq 1$,

$$
\begin{aligned}
& \left|\mathbf{k} C_{k}^{n}(t) S_{k}(0, t ; \mathbf{x})^{T} \boldsymbol{\zeta}_{k}^{T}\right| \\
\leq & \sum_{j=2}^{\infty} \frac{1}{j !}\left|\mathbf{k}\left\{\left(\frac{\beta_{G}}{\sqrt{n}} \int_{0}^{t} V_{n}(s) d s\right)^{j}\left(B_{k}^{j}\right)^{T}\right\} S_{k}(0, t ; \mathbf{x})^{T} \boldsymbol{\zeta}_{k}^{T}\right|
\end{aligned}
$$


For $j \geq 1$, the maximum element of $S_{k}(0, t ; \mathbf{x}) B_{k}^{j}$ is less than or equal to $(2 k)^{j}$ since $S_{k}(0, t ; \mathbf{x})$ is a stochastic matrix. Therefore

$$
\left|\mathbf{k}\left(B_{k}^{j}\right)^{T} S_{k}(0, t ; \mathbf{x})^{T} \boldsymbol{\zeta}_{k}^{T}\right| \leq k(2 k)^{j}=2^{j} k^{j+1} .
$$

Thus if $A_{n}(t)=2 \beta_{G}\left|\int_{0}^{t} V_{n}(s) d s\right|$, then by Theorem 5.1, $\mathbb{P}\left(A_{n}(t)<\infty\right)=1$

$$
\begin{aligned}
& \sqrt{n} \sum_{k=1}^{\infty} \omega_{k} \sum_{j=2}^{\infty}\left|\frac{1}{j !}\left(\frac{\beta_{G}}{\sqrt{n}} \int_{0}^{t} V_{n}(s) d s\right)^{j}\right| 2^{j} k^{j+1} \\
\leq & \sqrt{n} \sum_{k=1}^{\infty} k \omega_{k}\left|\exp \left(k \frac{1}{\sqrt{n}} A_{n}(t)\right)-1-k \frac{1}{\sqrt{n}} A_{n}(t)\right|
\end{aligned}
$$

Then since there exists $a>0$, such that $\mathbb{E}\left[e^{a H}\right]<\infty$, it is straightforward to show that the righthand-side of (5.11) converges in probability to 0 as $n \rightarrow \infty$.

Thus for all $t \geq 0, \epsilon_{n}^{2}(t) \stackrel{p}{\longrightarrow} 0$ as $n \rightarrow \infty$ and the Lemma follows.

For $t \geq 0$, let

$$
r(t ; \mathbf{x})=\sum_{k=1}^{\infty} \omega_{k} \mathbf{k} B_{k}^{T} S_{k}(0, t ; \mathbf{x})^{T} \boldsymbol{\zeta}_{k}^{T}
$$

It follows that $r(t ; \mathbf{x})<\infty$ since $\mathbb{E}\left[H^{2}\right]<\infty$.

Theorem 5.2. Suppose that there exists a $>0$, such that $\mathbb{E}[\exp (a H)]<\infty$. Then for all $T \geq 0, V_{n} \Rightarrow V$ as $n \rightarrow \infty$ on $[0, T]$ where $V$ satisfies

$$
V(t)=Y(t ; \mathbf{x})+\beta_{G} r(t ; \mathbf{x}) \int_{0}^{t} V(s) d s
$$

Proof. Firstly, note that, by Lemma 5.5, for all $t \geq 0$

$$
V_{n}(t)=Y_{n}\left(t ; \mathbf{X}_{n}\right)+\epsilon_{n}^{1}(t)+\epsilon_{n}^{2}(t)+\beta_{G} r(t ; \mathbf{x}) \int_{0}^{t} V_{n}(s) d s .
$$

For $m \geq 0$ and $0 \leq s \leq t$, define $C_{m}(s, t ; \mathbf{x})$ as follows. Let $C_{0}(s, t ; \mathbf{x})=1$ and for $m \geq 1$,

$$
C_{m}(s, t ; \mathbf{x})=\int_{s}^{t} \beta_{G} r(u ; \mathbf{x}) C_{m-1}(u, t ; \mathbf{x}) d u .
$$

Following both [17] and [13], if we let

$$
K(s, t ; \mathbf{x})=\sum_{m=0}^{\infty} C_{m}(s, t ; \mathbf{x}),
$$

then for all $0 \leq s \leq t, K(s, t ; \mathbf{x})<\infty$, and

$V_{n}(t)=Y_{n}\left(t ; \mathbf{X}_{n}\right)+\epsilon_{n}^{1}(t)+\epsilon_{n}^{2}(t)+\beta_{G} r(t ; \mathbf{x}) \int_{0}^{t} K(s, t ; \mathbf{x})\left\{Y_{n}\left(s ; \mathbf{X}_{n}\right)+\epsilon_{n}^{1}(s)+\epsilon_{n}^{2}(s)\right\} d s$. 
Then $J: D \rightarrow D$ defined by

$$
J y(t)=y(t)+\beta_{G} r(t ; \mathbf{x}) \int_{0}^{t} K(s, t ; \mathbf{x}) y(s) d s
$$

is continuous. Also $V_{n}(t)=J\left(Y_{n}+\epsilon_{n}^{1}+\epsilon_{n}^{2}\right)$ and $V(t)=J(Y)$. Therefore by the continuous mapping theorem (Theorem 25.7, Corollary 1 on page 288, [7]), $V_{n} \Rightarrow V$ as $n \rightarrow \infty$ on $[0, T]$.

\section{References}

[1] Ball, F. (1999). Stochastic and deterministic models for SIS epidemics among a population partitioned into households. Math. Biosci. 156 41-67.

[2] Ball, F. and Lyne, O. (2001) Stochastic multitype SIR epidemics among a population partitioned into households. Adv. Appl. Prob. 33, 99-123.

[3] Ball, F., Mollison, D. and Scalia-Tomba, G. (1997). Epidemics with two levels of mixing. Ann. Appl Probab. 7 46-89.

[4] Ball, F. and Neal, P. (2002) A general model for stochastic SIR epidemics with two levels of mixing. Math. Biosci. 180, 73-102.

[5] Becker, N. and Dietz, K. (1995) The effect of the household distribution on transmission and control of highly infectious diseases. Math. Biosci. 127 207-219.

[6] Billingsley, P. (1968). Convergence of Probability Measures. Wiley, New York.

[7] Billingsley, P. (1979). Probability and Measure. Wiley, New York.

[8] Clancy, D. and Pollett, P. (2003). A note on quasi-stationary distributions of birthdeath processes and the SIS logistic epidemic. J. Appl. Prob. 40 821-825.

[9] Ghoshal, G. Sander, L. and Sokolov, I. (2004). SIS epidemics with household structure: the self-consistent field method. Math. Biosci. 190 71-85.

[10] Kryscio, R. and Lefèrve, C. (1989). On the extinction of the SIS stochastic logistic epidemic. J. Appl. Prob. 26 685-694.

[11] Kurtz, T. (1970). Solutions of ordinary differential equations as limits of pure jump Markov processes. J. Appl. Prob. 7 49-58. 
[12] Kurtz, T. (1971). Limit theorems for sequences of jump Markov processes approximating ordinary differential processes. J. Appl. Prob. 8 344-356.

[13] Neal, P. (2005a). Asymptotic Gaussian approximations for open population epidemic models. Submitted to Stochastic Process. Appl.

[14] Neal, P. (2005b). Multitype randomised Reed-Frost epidemics and epidemics upon random graphs. Submitted to Annals Appl. Prob.

[15] Rogers, L. and Williams, D. (1994). Diffusions, Markov Processes and Martingales: Volume 1 Foundations. Wiley, New York.

[16] Ross, R. (1915) Some a priori pathometric equations. Br. Med. J. 1 546-547.

[17] Solomon, W. (1987). Representation and approximation of large population age distributions using Poisson random measures. Stochastic Process. Appl. 26, 237-255. 